\title{
J-PAS: forecasts on interacting vacuum energy models
}

\author{
V. Salzano, ${ }^{1,}$ C. Pigozzo, ${ }^{2}$ M. Benetti, ${ }^{3,4}$ H. A. Borges, ${ }^{2}$ R. von Marttens, ${ }^{5}$ S. Carneiro, ${ }^{2}$ J. S. Alcaniz, ${ }^{5}$ \\ J. C. Fabris, ${ }^{6}$ S. Tsujikawa, ${ }^{7}$ N. Benítez, ${ }^{8}$ S. Bonoli, $,{ }^{9},{ }^{10} 11$ A. J. Cenarro, ${ }^{12}$ D. Cristóbal-Hornillos, ${ }^{12}$ \\ R. A. Dupke, ${ }^{5,13}$ A. Ederoclite, ${ }^{14}$ C. López-Sanjuan, ${ }^{12}$ A. Marín-Franch, ${ }^{12}$ V. Marra,,${ }^{15,16,17}$ \\ M. Moles, ${ }^{12}$ C. Mendes de Oliveira, ${ }^{14}$ L. Sodré Jr,${ }^{14} \mathrm{~K}$. Taylor, ${ }^{18} \mathrm{~J}$. Varela, ${ }^{12}$ and H. Vázquez Ramió ${ }^{12}$ \\ ${ }^{1}$ Institute of Physics, University of Szczecin, Wielkopolska 15, 70-451 Szczecin, Poland \\ ${ }^{2}$ Instituto de Física, Universidade Federal da Bahia, 40210-340, Salvador, BA, Brazil \\ ${ }^{3}$ Dipartimento di Fisica "E. Pancini", Università di Napoli "Federico II", Via Cinthia, I-80126, Napoli, Italy \\ ${ }^{4}$ Istituto Nazionale di Fisica Nucleare (INFN), sez. di Napoli, Via Cinthia 9, I-80126 Napoli, Italy \\ ${ }^{5}$ Departamento de Astronomia, Observatório Nacional, Rio de Janeiro, 20921-400, RJ, Brazil \\ ${ }^{6}$ Núcleo Cosmo-ufes \& Departamento de Física, Universidade Federal do Espírito Santo, 29075-910, Vitória, ES, Brazil \\ ${ }^{7}$ Department of Physics, Waseda University, 3-4-1 Okubo, Shinjuku, Tokyo 169-8555, Japan \\ ${ }^{8}$ Instituto de Astrofísica de Andalucía (CSIC), Glorieta de la Astronomía s/n, Granada, 18008, Spain \\ ${ }^{9}$ Centro de Estudios de Física del Cosmos de Aragón (CEFCA), Plaza San Juan, 1, E-44001, Teruel, Spain \\ ${ }^{10}$ Donostia International Physics Center (DIPC), Manuel Lardizabal Ibilbidea, 4, San Sebastián, Spain \\ ${ }^{11}$ Ikerbasque, Basque Foundation for Science, E-48013 Bilbao, Spain \\ ${ }^{12}$ Centro de Estudios de Física del Cosmos de Aragón (CEFCA), \\ Unidad Asociada al CSIC, Plaza de San Juan, 1, E-44001, Teruel, Spain \\ ${ }^{13}$ Department of Astronomy, University of Michigan, 311 West Hall, 1085 South University Ave., Ann Arbor, USA \\ ${ }^{14}$ Departamento de Astronomia, Instituto de Astronomia, \\ Geofísica e Ciências Atmosféricas, Universidade de São Paulo, \\ R. do Matão 1226, São Paulo, SP 05508-090, Brazil \\ ${ }^{15}$ Núcleo de Astrofísica e Cosmologia \& Departamento de Física, \\ Universidade Federal do Espírito Santo, 29075-910, Vitória, ES, Brazil \\ ${ }^{16}$ INAF - Osservatorio Astronomico di Trieste, via Tiepolo 11, 34131 Trieste, Italy \\ ${ }^{17}$ IFPU - Institute for Fundamental Physics of the Universe, via Beirut 2, 34151, Trieste, Italy \\ ${ }^{18}$ Instruments4, 4121 Pembury Place, La Cañada-Flintridge, Ca 91011, USA
}

(Dated: February 15, 2021)

\begin{abstract}
The next generation of galaxy surveys will allow us to test some fundamental aspects of the standard cosmological model, including the assumption of a minimal coupling between the components of the dark sector. In this paper, we present the Javalambre Physics of the Accelerated Universe Astrophysical Survey (J-PAS) forecasts on a class of unified models where cold dark matter interacts with a vacuum energy, considering future observations of baryon acoustic oscillations, redshift-space distortions, and the matter power spectrum. After providing a general framework to study the background and linear perturbations, we focus on a concrete interacting model without momentum exchange by taking into account the contribution of baryons. We compare the J-PAS results with those expected for DESI and Euclid surveys and show that J-PAS is competitive to them, especially at low redshifts. Indeed, the predicted errors for the interaction parameter, which measures the departure from a $\Lambda C D M$ model, can be comparable to the actual errors derived from the current data of cosmic microwave background temperature anisotropies.
\end{abstract}




\section{INTRODUCTION}

The quest to uncover the origin of the underlying cause of late-time cosmic acceleration is one of the most important topics in cosmology [1-4]. In the context of the $\Lambda$-Cold-Dark-Matter $(\Lambda \mathrm{CDM})$ model [5, 6], gravity is described by Einstein's General Relativity in the presence of the cosmological constant $\Lambda$ and non-relativistic dark matter. Although the $\Lambda \mathrm{CDM}$ model is treated as a standard cosmological paradigm, the origins of the dark sector are largely unknown. Moreover, the recent observations have shown that, in the $\Lambda \mathrm{CDM}$ model, there are tensions for today's Hubble constant $H_{0}$ between the measurements of Cosmic Microwave Background (CMB) temperature anisotropies [7] and its direct measurements at low redshifts [8, 9].

Additionally, a basic hypothesis of standard cosmology is that non-relativistic matter, which forms galaxies and clusters, obeys the continuity equation by its own. This conserved property holds if dark energy, the mechanism behind the late-time acceleration, is a non-interacting vacuum energy $\Lambda$. If CDM interacts with $\Lambda$, the vacuum energy can be time-dependent [10-22]. Although the presence of $\Lambda$ does not give rise to any dynamical degrees of freedom, the modified background evolution induced by the coupling with CDM changes the cosmic distance to sources. Hence the interacting vacuum energy scenario can be observationally distinguished from the uncoupled $\Lambda$ CDM model [23].

In interacting vacuum energy models where the coupling is phenomenologically chosen to realize a desired background evolution with late-time cosmic acceleration, it is non-trivial to deal with the dynamics of inhomogeneous perturbations in a consistent way. [19] addressed this issue by considering the vacuum-energy perturbation $\delta \Lambda$ induced by the energy or momentum exchanges with CDM. Reflecting the fact that the four velocity of vacuum energy is undetermined, we need to make an assumption that $\delta \Lambda$ is related to other perturbations. One way is to assume that $\Lambda$ depends on the CDM density $\rho_{c}$ [19], in which case $\delta \Lambda$ has a relation with the CDM perturbation $\delta \rho_{c}$. The other possible way is to consider the case in which either energy or momentum exchange between $\Lambda$ and CDM is absent [24]. In this paper we will provide a general theoretical framework of interacting vacuum energy with CDM by taking into account baryons, but for the purpose of the J-PAS forecast, we will choose a concrete model in which the momentum exchange is absent.

Without restricting the origin of late-time cosmic acceleration to the cosmological constant, there are also other phenomenological approaches to dynamical dark energy models coupled to CDM in which the dark energy equation of state $w_{\mathrm{DE}}$ deviates from - 1 [25. 45]. Numerous observational data including CMB, supernovae type Ia (SNeIa), and Baryon Acoustic Oscillations (BAO) have been used to place constraints on such interacting models (see e.g., [46] and references therein). In particular, it was shown that the presence of couplings between dark energy and CDM can alleviate the observational tension of $H_{0}$ present in the $\Lambda \mathrm{CDM}$ model [47-56]. There are also dynamical interacting models of dark energy with some concrete Lagrangians of scalar fields or vector fields [57.-72], in which case it is possible to reduce observational tensions of $\sigma_{8}$. In interacting models of dark perfect fluids with a momentum exchange, it was recently shown that the observational tension of the $\Lambda$ CDM model can be also eased [73-75].

To place observational constraints on interacting models of dark energy, the next generation of galaxy surveys, such as DESI [76], Euclid [77], SKA [78] and Javalambre Physics of the Accelerated Universe Astrophysical Survey (J-PAS) [79, 80], will play major roles. In these models, not only the background evolution but also the dynamics of cosmological perturbations is modified in comparison to the $\Lambda$ CDM. With high-precision observational data, there will be a chance of detecting the signature of dark sector interactions, in spite of dark degeneracy mentioned in Refs. [81-86]. In this paper, we consider the simple interacting vacuum energy model mentioned above and discuss expected cosmological impacts extracted from J-PAS [79, 80] measurements. It is straightforward to extend the analysis to more general dynamical coupled dark energy models, but we will leave it for future work due to their complexities.

Our purpose in this paper is twofold. First, we study the implications of J-PAS on a concrete interacting model characterized by a coupling constant $\alpha$ (defined in Sec.V). Second, we compare the J-PAS forecast results with those expected from the DESI [76] and Euclid [77] surveys. For two configurations of area, i.e., $4000 \mathrm{deg}^{2}$ and $8500 \mathrm{deg}^{2}$, we consider the J-PAS information on BAO, Redshift-Space Distortions (RSD), and the full matter power spectrum (PS), and perform a Fisher matrix forecast on the parameter $\alpha$, which also quantifies a possible deviation from the $\Lambda \mathrm{CDM}$ cosmology $(\alpha=0)$. In order to improve the accuracy of estimates, we also perform a multi-tracer analysis and compare all J-PAS forecasts with those expected by the DESI and Euclid surveys. We find that J-PAS will be able to measure the interaction parameter with good sensitivity, and will provide the best constraints on interaction in the redshift range $z \lesssim 0.5$, thanks to the large number of emission line galaxies detectable in that redshift range.

The outline of this paper is as follows. Section II briefly introduces the main characteristics of the J-PAS. General aspects of interacting vacuum energy including the background equations of motion are discussed in Sec. III. In Sec. IV] we derive the perturbation equations in a gauge-ready form and discuss different choices of gauges. In Sec. $V$, we present a concrete model of interacting vacuum energy and study the background and perturbation dynamics taking into account baryon perturbations. Sec.VI presents our Fisher matrix analysis, whereas Sec. VII discusses our main results, including a comparison with forecasts for the DESI and Euclid surveys. We summarize our main conclusions in Sec.VIII. 


\section{J-PAS}

The J-PAS [79, 80] is a spectro-photometric, ground-based imaging survey that will observe $8500 \mathrm{deg}^{2}$ of the northern sky. The survey will be conducted at the Observatorio Astrofísico de Javalambre, a site on top of Pico del Buitre, a summit about $\sim 2000 \mathrm{~m}$ high above sea level at the Sierra de Javalambre (Spain).

The survey will be carried out in a seven-year observing program at a dedicated $2.5 \mathrm{~m}$ telescope, the Javalambre Survey Telescope (JST/T250), equipped with a 1.2 Gigapixel camera (JPCam) with a large field of view of $4.2 \mathrm{deg}^{2}$, and will incorporate a 54 narrow- and 4 broad-band filter set covering the optical range. J-PAS will measure positions and redshifts for dozens of millions of Luminous Red Galaxies (LRG), Emission Line Galaxies (ELG) and millions of Quasars (QSO), with an expected photometric redshift precision of $\sigma(z)=0.003(1+z)$, where $z$ is the redshift. Moreover, given its high photo- $z$ precision, J-PAS will detect nearly a hundred thousand clusters of galaxies up to the redshift $z \sim 1$ and several times more groups of galaxies. They can be used to improve the constraints on cosmological parameters not just through the mass function but also through high-precision definition of the cosmic web. For more details on J-PAS, we refer the reader to [79, 80].

\section{GENERAL ASPECTS OF INTERACTING VACUUM ENERGY}

We consider cold dark matter interacting with a vacuum energy $\Lambda$ [10-21]. The CDM is described by a pressureless perfect fluid given by the energy-momentum tensor

$$
T_{c}^{\mu v}=\rho_{c} u^{\mu} u^{v}
$$

where $\rho_{c}$ is the CDM density, and $u^{\mu}$ is the four velocity obeying the relation $u_{\mu} u^{\mu}=-1$. The energy-momentum tensor of vacuum is given by

$$
T_{\Lambda}^{\mu v}=-\Lambda g^{\mu v},
$$

where $g^{\mu \nu}$ is the metric tensor. The vacuum energy density $\hat{\rho}$ and pressure $\hat{P}$ satisfy $\hat{\rho}=-\hat{P}=\Lambda$, i.e., $\hat{\rho}+\hat{P}=0$, so its four velocity $\hat{u}_{\mu}$ is undefined. Unlike CDM, the vacuum energy does not act as a dynamical degree of freedom. Taking the covariant derivative of Eq. (2), it follows that

$$
T_{\Lambda ; v}^{\mu v}=-Q^{\mu}
$$

where

$$
Q^{\mu}=\Lambda_{, v}{ }^{\mu \nu}
$$

The semicolon and colon represent the covariant and partial derivatives, respectively. If there is the energy or momentum transfer between CDM and vacuum energy, we have $Q^{\mu} \neq 0$ and hence the vacuum energy becomes inhomogeneous in spacetime [19].

We assume that the total energy-momentum tensor $T^{\mu \nu}=T_{c}^{\mu \nu}+T_{\Lambda}^{\mu \nu}$ satisfies the continuity equation $T_{; v}^{\mu \nu}=0$. On using Eq. (3), the CDM sector obeys

$$
T_{c ; v}^{\mu \nu}=+Q^{\mu}
$$

We decompose $Q^{\mu}$ in the form

$$
Q^{\mu}=Q u^{\mu}+q^{\mu}
$$

where $q^{\mu}$ satisfies

$$
q^{\mu} u_{\mu}=0
$$

Substituting Eq. (6) into Eq. (4) and multiplying it by $u_{\mu}$, it follows that

$$
Q=-\Lambda_{,} u^{\nu}
$$

On using Eqs. (4), (6), and (8), we obtain

$$
q^{\mu}=\Lambda_{, v}\left(u^{\mu} u^{v}+g^{\mu v}\right)
$$


The quantities $Q$ and $q^{\mu}$ represent the energy transfer and momentum transfer, respectively. Indeed, the energy exchange between two dark components is explicit by taking the products of $u_{\mu}$ in Eqs. (3) and (5) [70, 71], such that

$$
\begin{aligned}
& u_{\mu} T_{\Lambda ; v}^{\mu v}=+Q, \\
& u_{\mu} T_{c ; v}^{\mu \nu}=-Q .
\end{aligned}
$$

On the right hand-side of these equations, the terms associated with the momentum transfer vanish because of the condition (7).

Let us consider the spatially flat Friedmann-Lamaître-Robertson-Walker (FLRW) spacetime given by the line element

$$
\mathrm{d} s^{2}=-\mathrm{d} t^{2}+a^{2}(t) \delta_{i j} \mathrm{~d} x^{i} \mathrm{~d} x^{j}
$$

where $a$ is the scale factor which depends on the cosmic time $t$. On this background, the CDM four velocity can be chosen as $u^{\mu}=(1,0,0,0)$ and hence the momentum transfer 9 vanishes. Then, from Eqs. (10) and [11, it follows that

$$
\begin{aligned}
\dot{\Lambda} & =-Q, \\
\dot{\rho}_{c}+3 H \rho_{c} & =Q,
\end{aligned}
$$

where a dot represents a derivative with respect to $t$, and $H \equiv \dot{a} / a$ is the Hubble expansion rate. In the gravity sector we consider general relativity with the Einstein equation

$$
G^{\mu v}=8 \pi G\left(T_{c}^{\mu v}+T_{\Lambda}^{\mu v}\right)
$$

where $G^{\mu \nu}$ is the Einstein tensor, and $G$ is the gravitational constant. We neglect the contribution of baryons to the right hand-side of Eq. (15), but we will include it in Secs. V A V C The background equations of motion following from Eq. (15) are

$$
\begin{aligned}
3 H^{2} & =8 \pi G\left(\rho_{c}+\Lambda\right), \\
\dot{H} & =-4 \pi G \rho_{c},
\end{aligned}
$$

where Eq. (17) can be also derived by taking the time derivative of Eq. (16) and employing Eqs. (13) and (14).

\section{COSMOLOGICAL PERTURBATIONS}

Let us consider scalar perturbations on the flat FLRW background 12 . The perturbed line element, which contains four metric perturbations $A, B, \psi$, and $E$, is given by [87]

$$
\mathrm{d} s^{2}=-(1+2 A) \mathrm{d} t^{2}+2 a \partial_{i} B \mathrm{~d} t \mathrm{~d} x^{i}+a^{2}\left[(1-2 \psi) \delta_{i j}+2 \partial_{i} \partial_{j} E\right] \mathrm{d} x^{i} \mathrm{~d} x^{j},
$$

where we used the notation $\partial_{i} \equiv \partial / \partial x^{i}$. Since we are interested in the evolution of linear perturbations, we neglect the terms higher than the first order in the following discussion.

For CDM, the energy density is decomposed into the background and perturbed parts, as $\rho_{c}=\bar{\rho}_{c}+\delta \rho_{c}$. The CDM four velocity is defined by $u^{\mu}=\mathrm{d} x^{\mu} / \mathrm{d} \tau$, where $\tau$ is the proper time satisfying $\mathrm{d} \tau=(1+A) \mathrm{d} t$ for the perturbed line element $(18)$. We express the spatial component of $u^{\mu}$ as $u^{i}=a^{-1} \partial^{i} v$, where $v$ is the scalar velocity potential. Then, the CDM four velocities with upper and lower indices are expressed as

$$
u^{\mu}=\left(1-A, a^{-1} \partial^{i} v\right), \quad u_{\mu}=\left(-1-A, \partial_{i} \theta\right)
$$

where

$$
\theta \equiv a(v+B)
$$

The four velocity of vacuum energy, $\hat{u}_{\mu}$, can be defined by using the energy-momentum flow (4), such that

$$
\hat{u}_{\mu}=-\frac{\Lambda_{, \mu}}{\left|\Lambda_{, v} \Lambda^{, v}\right|^{1 / 2}},
$$

which obeys the relation $\hat{u}_{\mu} \hat{u}^{\mu}=-1$ for the time-like flow $\left(\Lambda_{, v} \Lambda^{, v}<0\right)$. By introducing the vacuum energy velocity potentials $\hat{v}$ and $\hat{\theta}$, as $\hat{u}^{i}=a^{-1} \partial^{i} \hat{v}$ and $\hat{\theta}=a(\hat{v}+B)$, we can express $\hat{u}^{\mu}$ in a fashion analogous to Eq. [19]:

$$
\hat{u}^{\mu}=\left(1-A, a^{-1} \partial^{i} \hat{v}\right), \quad \hat{u}_{\mu}=\left(-1-A, \partial_{i} \hat{\theta}\right) .
$$

The vacuum energy has a perturbation $\delta \Lambda$, which is induced by the interaction with CDM. From Eq. 21) the spatial component of $\hat{u}_{\mu}$ is $\hat{u}_{i}=-\partial_{i} \delta \Lambda / \dot{\Lambda}$, so the comparison with the second of Eq. 22, gives

$$
\delta \Lambda=-\dot{\Lambda} \hat{\theta}=Q \hat{\theta}
$$

where we used Eq. 13. This shows that $\delta \Lambda$ is directly related to the velocity potential of vacuum energy. 


\section{A. Perturbation equations}

We split the scalar quantity $Q$ into the background and perturbed parts, as $Q=\bar{Q}+\delta Q$. In the following, we omit a bar from the background quantities. Then, the linear perturbation equations following from Eqs. (10) and (11) are given, respectively, by [19]

$$
\begin{aligned}
& \dot{\delta \Lambda}=-\delta Q-Q A, \\
& \dot{\delta \rho_{c}}+3 H \delta \rho_{c}-3 \rho_{c} \dot{\psi}+\rho_{c} \frac{\nabla^{2}}{a^{2}}\left(\theta+a^{2} \dot{E}-a B\right)=\delta Q+Q A,
\end{aligned}
$$

where $\nabla^{2}$ is the three-dimensional Laplacian. The perturbation of spatial components of $Q^{\mu}$ in Eq. (6) is expressed as

$$
\delta Q^{i}=Q u^{i}+\delta q^{i}=\partial^{i}\left(a^{-1} Q v+a^{-2} f\right),
$$

where a scalar function $f$ is related to the perturbation of $q_{i}$ in the form

$$
\delta q_{i}=\partial_{i} f .
$$

The $\mu=i$ components of Eqs. (3) and (5) give the Euler equations

$$
\begin{aligned}
\delta \Lambda & =f+Q \theta, \\
\rho_{c} \dot{\theta}+\rho_{c} A & =f .
\end{aligned}
$$

Equation (29) means that the CDM feels the force $f$ through the interaction with vacuum energy. From Eqs. (23) and (28), we obtain

$$
f=Q(\hat{\theta}-\theta),
$$

which does not vanish for $\hat{\theta} \neq \theta$. If there is the difference between the four velocities of CDM and vacuum energy, there is the momentum transfer which affects the evolution of CDM velocity potential.

We define the CDM density contrast, as

$$
\delta_{c} \equiv \frac{\delta \rho_{c}}{\rho_{c}} .
$$

From Eqs. 24-25) and 28)-29, the two variables $\delta_{c}$ and $\theta$ satisfy the first-order differential equations

$$
\begin{aligned}
\dot{\delta}_{c}-3 \dot{\psi}+\frac{\nabla^{2}}{a^{2}}(\theta+a \sigma) & =-\frac{\dot{\delta \Lambda}-\dot{\Lambda} \delta_{c}}{\rho_{c}}, \\
\dot{\theta}+A & =\frac{\delta \Lambda+\dot{\Lambda} \theta}{\rho_{c}},
\end{aligned}
$$

where

$$
\sigma \equiv a \dot{E}-B
$$

The linearly perturbed Einstein equations are given by $\delta G^{\mu v}=8 \pi G\left(\delta T_{m}^{\mu v}+\delta T_{\Lambda}^{\mu v}\right)$, whose (00), $(0 i)$, and $(i j)(i \neq j)$ components lead to

$$
\begin{aligned}
3 H(\dot{\psi}+H A)-\frac{\nabla^{2}}{a^{2}}(\psi+a H \sigma) & =-4 \pi G\left(\rho_{c} \delta_{c}+\delta \Lambda\right), \\
\dot{\psi}+H A & =-4 \pi G \rho_{c} \theta, \\
a(\dot{\sigma}+2 H \sigma)-A+\psi & =0 .
\end{aligned}
$$

Thus, we derived the full set of linear perturbation equations (32)- 33 and 35]- 377 without choosing particular gauges. The vacuum fluctuation $\delta \Lambda$, which is related to its four velocity $\hat{\theta}$ as Eq. 23 , can be determined by specifying a model of interacting vacuum energy [19]. If $\hat{\theta}=\theta$, i.e., $\delta \Lambda=-\dot{\Lambda} \theta$, the right hand-side of Eq. 33 vanishes, with $-\dot{\Lambda}=\ddot{\Lambda} \theta+\dot{\Lambda} \dot{\theta}$ on the right hand-side of Eq. (32). In this case, there is no momentum transfer $\left(\delta q_{i}=0\right)$ between CDM and vacuum energy. This is an example where the perturbation equations of motion are closed. 
We introduce the following gauge-invariant gravitational potentials [87],

$$
\Psi=A-\frac{\mathrm{d}}{\mathrm{d} t}(a \sigma), \quad \Phi=\psi+a H \sigma .
$$

Then, from Eq. (37), we obtain

$$
\Psi=\Phi
$$

which shows the absence of an anisotropic stress.

Although we have not chosen particular gauges so far, there are residual gauge degrees of freedom to be fixed. In the following, we will consider two different gauge choices.

\section{B. Newtonian gauge}

Let us first choose the Newtonian gauge satisfying

$$
B=0, \quad E=0,
$$

under which $\sigma=0$. In this case, the gravitational potentials 38 are given by

$$
\Psi=A, \quad \Phi=\psi .
$$

On using the relation (39), Eqs. 32, 33, 35, and (36) reduce, respectively, to

$$
\begin{aligned}
\dot{\delta}_{c}-3 \dot{\Phi}+\frac{\nabla^{2}}{a^{2}} \theta & =-\frac{\dot{\delta} \Lambda-\dot{\Lambda} \delta_{c}}{\rho_{c}}, \\
\dot{\theta}+\Phi & =\frac{\delta \Lambda+\dot{\Lambda} \theta}{\rho_{c}}, \\
3 H(\dot{\Phi}+H \Phi)-\frac{\nabla^{2}}{a^{2}} \Phi & =-4 \pi G\left(\rho_{c} \delta_{c}+\delta \Lambda\right), \\
\dot{\Phi}+H \Phi & =-4 \pi G \rho_{c} \theta .
\end{aligned}
$$

For a given physical model relating $\delta \Lambda$ with other perturbations, these equations can be solved for $\delta_{c}, \theta, \Phi$, and $\delta \Lambda$.

\section{Synchronous gauge}

The synchronous gauge is characterized by the conditions

$$
A=0, \quad B=0,
$$

under which $\sigma=a \dot{E}$. The gravitational potentials reduce to

$$
\Psi=-\frac{\mathrm{d}}{\mathrm{d} t}\left(a^{2} \dot{E}\right), \quad \Phi=\psi+a^{2} H \dot{E} .
$$

We introduce the following combination

$$
h \equiv 2 \nabla^{2} E-6 \psi
$$

Then, Eqs. (32), 33), 35, and 36 yield

$$
\begin{aligned}
\dot{\delta}_{c}+\frac{1}{2} \dot{h}+\frac{\nabla^{2}}{a^{2}} \theta & =-\frac{\dot{\delta} \Lambda-\dot{\Lambda} \delta_{c}}{\rho_{c}}, \\
\dot{\theta} & =\frac{\delta \Lambda+\dot{\Lambda} \theta}{\rho_{c}}, \\
\frac{\nabla^{2}}{a^{2}} \psi+\frac{1}{2} H \dot{h} & =4 \pi G\left(\rho_{c} \delta_{c}+\delta \Lambda\right), \\
\dot{\psi} & =-4 \pi G \rho_{c} \theta .
\end{aligned}
$$


Equation (37), which is equivalent to Eq. 39], gives the differential equation for $E$, as

$$
a^{2}(\ddot{E}+3 H \dot{E})+\psi=0 .
$$

Exerting the operator $\nabla^{2}$ on Eq. 53, and using Eqs. 48, , 51, and 52, the perturbation $h$ obeys

$$
\ddot{h}+2 H \dot{h}+8 \pi G\left(\rho_{c} \delta_{c}-3 \rho_{c} \dot{\theta}+\delta \Lambda+3 \dot{\Lambda} \theta\right)=0 .
$$

For a given relation of $\delta \Lambda$ with other perturbations, we can integrate Eqs. [49, (50), (52), (53), and (54) to solve for $\delta_{c}, \theta, \psi, E$, and $h$.

\section{CONCRETE INTERACTING MODEL}

Let us consider a concrete interacting model of vacuum energy and dark matter. For perturbations, this amounts to giving an explicit relation between $\delta \Lambda$ and other perturbations. We study the case in which there is no momentum transfer in Eq. (6), i.e.,

$$
q^{\mu}=0
$$

under which the interaction is restricted to be $Q^{\mu}=Q u^{\mu}$. In this case we have $\delta q_{i}=\partial_{i} f=0$, so the force $f=Q(\hat{\theta}-\theta)$ exerting on the $\mathrm{CDM}$ perturbation vanishes, i.e., $\hat{\theta}=\theta$. Then, from Eq. 23 , the perturbation $\delta \Lambda$ is related to $\theta$, as

$$
\delta \Lambda=-\dot{\Lambda} \theta=Q \theta .
$$

Under this condition the right hand side of the Euler Eq. 33 is zero, while this is not the case for the terms on the right hand side of the continuity Eq. (32).

Let us derive the perturbation equation of motion for $\delta_{c}$ in the synchronous gauge. From Eq. 50, we obtain $\dot{\theta}=0$, i.e.,

$$
\theta=\theta\left(x^{i}\right),
$$

which means that $\theta$ does not depend on time. In Fourier space with the comoving wavenumber $k$, we can write Eq. (49) in the form

$$
\dot{\delta}_{c}+\frac{1}{2} \dot{h}-\frac{k^{2}}{a^{2}} \theta=-\frac{\dot{Q} \theta+Q \delta_{c}}{\rho_{c}},
$$

where we used Eq. (56). We take the time derivative of Eq. 58, and eliminate the term $2 H\left(k^{2} / a^{2}\right) \theta$ on account of Eq. 58). On using Eq. [54], the CDM density contrast obeys

$$
\ddot{\delta}_{c}+\left(2 H+\frac{Q}{\rho_{c}}\right) \dot{\delta}_{c}-\left[4 \pi G \rho_{c}+\frac{Q^{2}-(\dot{Q}+5 H Q) \rho_{c}}{\rho_{c}^{2}}\right] \delta_{c}+\left[8 \pi G Q+\frac{(\ddot{Q}+5 H \dot{Q}) \rho_{c}-\dot{Q} Q}{\rho_{c}^{2}}\right] \theta=0 .
$$

From Eq. 58, the term $\left(k^{2} / a^{2}\right) \theta$ is at most of the order $\dot{\delta}_{c} \lesssim H \delta_{c}$, and hence $\theta \lesssim a^{2} H \delta_{c} / k^{2}$. Moreover, from Eq. (14), the coupling $Q$ should be of order $Q \lesssim 3 H \rho_{c}$. Then, the ratio between the terms $8 \pi G Q \theta$ and $4 \pi G \rho_{c} \delta_{c}$ can be estimated as

$$
r \equiv \frac{8 \pi G Q \theta}{4 \pi G \rho_{c} \delta_{c}} \lesssim\left(\frac{a H}{k}\right)^{2} .
$$

For perturbations deep inside the Hubble radius $(k \gg a H)$, it follows that $r \ll 1$. Similarly, the other terms appearing as coefficients of $\theta$ in Eq. (59) are suppressed relative to the terms proportional to $\delta_{c}$. Then, for the modes $k \gg a H$, Eq. (59) is approximately given by

$$
\ddot{\delta}_{c}+\left(2 H+\frac{Q}{\rho_{c}}\right) \dot{\delta}_{c}-\left[4 \pi G \rho_{c}+\frac{Q^{2}-(\dot{Q}+5 H Q) \rho_{c}}{\rho_{c}^{2}}\right] \delta_{c} \simeq 0 .
$$

Actually, in view of the time independence of $\theta$ in the synchronous gauge, we can always set it zero, which turns Eq. (61) an exact result. Note, however, that the sub-horizon approximation is necessary to obtain the same equation in the Newtonian gauge. Although we have chosen the synchronous gauge, the analysis in the Newtonian gauge gives rise to the same equation as (61) for the modes deep inside the Hubble radius. Introducing the variables

$$
\Gamma_{c} \equiv \frac{Q}{\rho_{c}}, \quad \eta \equiv \int a^{-1} \mathrm{~d} t,
$$

we can rewrite Eq. (61) in the form

$$
\delta_{c}^{\prime \prime}+a\left(H+\Gamma_{c}\right) \delta_{c}^{\prime}-\left[4 \pi G a^{2} \rho_{c}-\left(a \Gamma_{c}\right)^{\prime}-a^{2} \Gamma_{c} H\right] \delta_{c} \simeq 0,
$$

where a prime represents the derivative with respect to $\eta$. The coupling $\Gamma_{c}$ modifies the growth rate of $\delta_{c}$ in comparison to the uncoupled case. 


\section{A. Inclusion of baryons}

So far, we have ignored the perturbation of baryons, but we would like to take it into account for the J-PAS forecast. We assume that baryons are coupled to neither vacuum energy nor dark matter. Then, the background baryon density $\rho_{b}$ obeys the continuity equation

$$
\dot{\rho}_{b}+3 H \rho_{b}=0
$$

with a vanishing pressure $\left(p_{b}=0\right)$.

Let us consider the synchronous gauge with the additional baryon density contrast $\delta_{b}$ and velocity field $\theta_{b}$. Then, the resulting continuity and Euler equations are given, respectively, by

$$
\begin{aligned}
\dot{\delta}_{b}+\frac{1}{2} \dot{h}+\frac{\nabla^{2}}{a^{2}} \theta_{b} & =0, \\
\dot{\theta}_{b} & =0,
\end{aligned}
$$

besides the perturbation Eqs. (49) and 50, for CDM. The perturbed Einstein Eqs. (51) and (52) are modified to

$$
\begin{aligned}
\frac{\nabla^{2}}{a^{2}} \psi+\frac{1}{2} H \dot{h} & =4 \pi G\left(\rho_{m} \delta_{m}+\delta \Lambda\right), \\
\dot{\psi} & =-4 \pi G\left(\rho_{c} \theta+\rho_{b} \theta_{b}\right),
\end{aligned}
$$

where

$$
\rho_{m} \delta_{m} \equiv \rho_{c} \delta_{c}+\rho_{b} \delta_{b}, \quad \rho_{m} \equiv \rho_{c}+\rho_{b},
$$

while Eq. (53) is unchanged. Following the same procedure as that explained in Sec. IV C and using Eq. 66, the perturbation $h$, which is defined in Eq. (48), obeys

$$
\ddot{h}+2 H \dot{h}+8 \pi G\left(\rho_{m} \delta_{m}-3 \rho_{c} \dot{\theta}+\delta \Lambda+3 \dot{\Lambda} \theta\right)=0 .
$$

Now, we focus on the case in which there is no momentum exchange between vacuum energy and dark matter. Since the condition 57 holds, we have $\dot{\theta}=0$ in Eq. 70 . For perturbations deep inside the Hubble radius, the terms $\delta \Lambda=Q \theta$ and $3 \dot{\Lambda} \theta=-3 Q \theta$ in Eq. 70, are neglected relative to $\rho_{c} \delta_{c}$, see Eq. 60. Then, Eq. 70 approximately reduces to

$$
\ddot{h}+2 H \dot{h}+8 \pi G \rho_{m} \delta_{m} \simeq 0 \text {. }
$$

Differentiating Eqs. (49) and (65) with respect to $t$ and taking the similar procedure to that explained in Sec. IV C, the CDM and baryon density contrasts in Fourier space obey the following differential equations

$$
\begin{aligned}
& \ddot{\delta}_{c}+\left(2 H+\frac{Q}{\rho_{c}}\right) \dot{\delta}_{c}-4 \pi G \rho_{m} \delta_{m}-\frac{Q^{2}-(\dot{Q}+5 H Q) \rho_{c}}{\rho_{c}^{2}} \delta_{c} \simeq 0, \\
& \ddot{\delta}_{b}+2 H \dot{\delta}_{b}-4 \pi G \rho_{m} \delta_{m} \simeq 0,
\end{aligned}
$$

which are valid for the modes $k \gg a H$. By using the variables $\Gamma_{c}$ and $\eta$ defined in Eq. 62, these equations can be expressed as

$$
\begin{gathered}
\delta_{c}^{\prime \prime}+a\left(H+\Gamma_{c}\right) \delta_{c}^{\prime}-4 \pi G a^{2} \rho_{m} \delta_{m}+\left[\left(a \Gamma_{c}\right)^{\prime}+a^{2} \Gamma_{c} H\right] \delta_{c} \simeq 0 \\
\delta_{b}^{\prime \prime}+a H \delta_{b}^{\prime}-4 \pi G a^{2} \rho_{m} \delta_{m} \simeq 0 .
\end{gathered}
$$

Since $\left|\rho_{c} \delta_{c}\right| \gg\left|\rho_{b} \delta_{b}\right|$, the baryon density contrast grows under the influence of gravitational potentials sourced mostly by the CDM density perturbation. There is no scale-dependence in Eqs. (74) and (75) governing the evolution of perturbations on sub-horizon scales, whose property is attributed to the absence of pressures for CDM and baryons. In our interacting theory, the dark energy pressure does not affect the sound speeds of CDM and baryons either.

We can also derive the perturbation equations of motion for the total density matter contrast $\delta_{m}$ defined in Eq. 69. On using the approximation similar to Eq. (60) for the modes deep inside the Hubble radius, we obtain

$$
\ddot{\delta}_{m}+\left(2 H+\frac{Q}{\rho_{m}}\right) \dot{\delta}_{m}-\left[4 \pi G \rho_{m}+\frac{Q^{2}-(\dot{Q}+5 H Q) \rho_{m}}{\rho_{m}^{2}}\right] \delta_{m} \simeq Q \frac{k^{2}}{a^{2}} \frac{\rho_{b}}{\rho_{m}^{2}}\left(\theta-\theta_{b}\right) .
$$


In terms of the conformal time $\eta$, this can be expressed as

$$
\delta_{m}^{\prime \prime}+a(H+\Gamma) \delta_{m}^{\prime}-\left[4 \pi G a^{2} \rho_{m}-(a \Gamma)^{\prime}-a^{2} \Gamma H\right] \delta_{m} \simeq Q k^{2} \frac{\rho_{b}}{\rho_{m}^{2}}\left(\theta-\theta_{b}\right)
$$

where

$$
\Gamma \equiv \frac{Q}{\rho_{m}} .
$$

The right hand side of Eq. (77) does not vanish for $\theta \neq \theta_{b}$. This means that the momentum exchange between CDM and baryons can affect the dynamics of $\delta_{m}$. Since both $\theta$ and $\theta_{b}$ are constants in time, this term vanishes for the initial condition $\theta=\theta_{b}$. In this case, Eq (77) reduces to the second-order differential equation of $\delta_{m}$.

\section{B. Background}

Let us consider a possible choice of the interacting function $Q$ to study the background cosmological evolution. We take into account the baryons whose background density $\rho_{b}$ obeys the continuity Eq. (64). The densities of vacuum energy and CDM satisfy Eqs. (13) and (14). In the presence of baryons, Eqs. (16) and (17) are modified to

$$
\begin{aligned}
3 H^{2} & =8 \pi G\left(\rho_{m}+\Lambda\right), \\
\dot{H} & =-4 \pi G \rho_{m},
\end{aligned}
$$

where $\rho_{m}=\rho_{c}+\rho_{b}$. For the interacting vacuum energy, we choose a function of the form [88]

$$
\Lambda=\frac{\sigma H^{-2 \alpha}}{8 \pi G}
$$

where $\sigma(>0)$ and $\alpha(>-1)$ are constants. From Eq. (79), there is the relation

$$
\sigma=3\left(1-\Omega_{m}\right) H_{0}^{2(\alpha+1)}
$$

where $\Omega_{m}=8 \pi G \rho_{m 0} /\left(3 H_{0}^{2}\right)$ is today's density parameter of total non-relativistic matter (the subscript " 0 " represents today's values). On using Eqs. (79), (80), and (81), the Hubble parameter obeys the differential equation

$$
\frac{\mathrm{d} H}{\mathrm{~d} z}=\frac{3 H}{2(1+z)}\left[1-\left(1-\Omega_{m}\right)\left(\frac{H}{H_{0}}\right)^{-2(\alpha+1)}\right],
$$

where $z=1 / a-1$ is the redshift, and we used the relation $\mathrm{d} z / \mathrm{d} t=-(1+z) H$. Integrating Eq. 83, with respect to $z$, we obtain

$$
H(z)=H_{0}\left[1-\Omega_{m}+\Omega_{m}(1+z)^{3(\alpha+1)}\right]^{1 /[2(\alpha+1)]},
$$

which satisfies $H=H_{0}$ at $z=0$. The decay rate 78 is given by

$$
\Gamma=-\frac{\dot{\Lambda}}{\rho_{m}}=\frac{\alpha \sigma H^{-2 \alpha-1}}{4 \pi G} \frac{\dot{H}}{\rho_{m}}=-\alpha \sigma H^{-2 \alpha-1}
$$

The non-interacting cosmological constant corresponds to $\alpha=0$, in which case $\Gamma=0$. For $\alpha<0$, the creation of CDM occurs through the positive coupling $\Gamma$. In particular, $\Gamma$ is constant for $\alpha=-1 / 2$. From 84 we observe that the ansatz 81, corresponds to a decomposed, non-adiabatic generalised Chaplygin gas [89. 96], which behaves like conserved matter at high redshifts and approaches a cosmological constant in the asymptotic future. The late-time non-adiabaticity prevents oscillations and instabilities in the power spectrum, contrary to what happens in adiabatic versions of the generalised Chaplygin gas [97-105]. This parametrization does not encompass all possible forms of interactions, but it can be used to search for signatures of the interacting vacuum energy in current and future observations.

The baryon density has the following redshift dependence,

$$
\rho_{b}(z)=\frac{3 H_{0}^{2}}{8 \pi G} \Omega_{b}(1+z)^{3},
$$



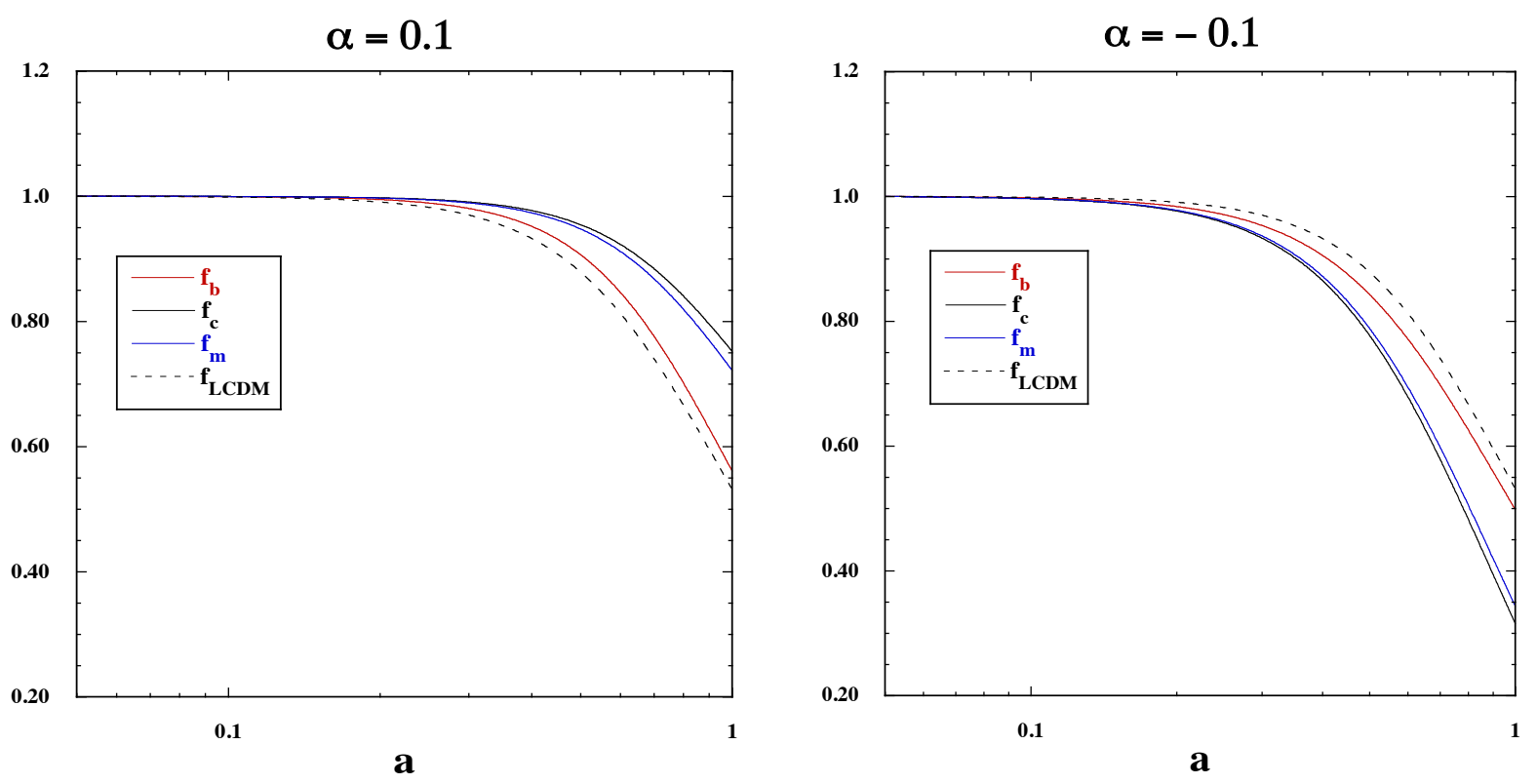

Figure 1. Evolution of $f_{b}, f_{c}$, and $f_{m}$ versus the scale factor $a$ for $\alpha=0.1$ (left) and $\alpha=-0.1$ (right), respectively. We also plot the evolution of $f_{b}\left(=f_{c}=f_{m}\right)$ in the $\Lambda$ CDM model as a dashed curve. Today's values of the density parameters are chosen to be $\Omega_{b}=0.05$ and $\Omega_{m}=0.32$.

where $\Omega_{b}=8 \pi G \rho_{b 0} /\left(3 H_{0}^{2}\right)$. On using Eqs. [80, , 84, and Eq. [86, the CDM density $\rho_{c}=\rho_{m}-\rho_{b}$ is given by the form

$$
\rho_{c}(z)=\frac{3 H_{0}^{2}}{8 \pi G}(1+z)^{3}\left[(1+z)^{3 \alpha} \Omega_{m}\left\{1-\Omega_{m}+\Omega_{m}(1+z)^{3(\alpha+1)}\right\}^{-\alpha /(\alpha+1)}-\Omega_{b}\right] .
$$

At high redshifts $(z \gg 1)$, it follows that

$$
\rho_{c}(z) \simeq \frac{3 H_{0}^{2}}{8 \pi G}(1+z)^{3}\left[\Omega_{m}^{1 /(\alpha+1)}-\Omega_{b}\right] .
$$

To avoid a negative CDM density, we require that $\rho_{c}(z)>0$. This condition translates to

$$
\alpha>\alpha_{c} \equiv-1+\frac{\ln \Omega_{m}}{\ln \Omega_{b}} .
$$

When $\Omega_{m}=0.32$ and $\Omega_{b}=0.05$, for example, we have $\alpha_{c}=-0.62$. At low redshifts around $z=0$, the expansion of Eq. 87p shows that, as long as $\Omega_{m}>\Omega_{b}$, the leading-order term of $\rho_{c}(z)$ is positive. For theoretical consistency, $\alpha$ should be in the range 89 .

\section{Growth rate of perturbations}

We define the functions describing the growth rates of CDM, baryons, and total matter, as

$$
f_{i}(a) \equiv \frac{\mathrm{d} \ln \delta_{i}(a)}{\mathrm{d} \ln a}=\frac{\dot{\delta}_{i}}{H \delta_{i}},
$$

where $i=b, c, m$, respectively. For sub-horizon perturbations, these functions are known by integrating Eqs. (74) and (75) together with the relation (69). In Fig. 11, we plot the evolution of $f_{b}, f_{c}$, and $f_{m}$ for $\alpha=0.1$ (left) and $\alpha=-0.1$ (right). We choose the initial conditions of density contrasts same as those in the $\Lambda$ CDM model $(\alpha=0)$, i.e., $f_{b}=1$ and $f_{c}=1$ at $a=0.05$. For the purpose of showing the evolution of $f_{i}$, it is sufficient to choose an arbitrary amplitude satisfying $\delta_{b}=\delta_{c}$.

In Fig. 11. the evolution of growth rates in the $\Lambda \mathrm{CDM}$ model is also shown, in which case $f_{b}=f_{c}=f_{m}$ due to the absence of interactions and the choice of same initial conditions for $\delta_{b}$ and $\delta_{c}$. At late times the interacting vacuum energy model with 
$\alpha>0$ leads to the value of $f_{c}$ larger than in the $\alpha=0$ case, while for $\alpha<0, f_{c}$ is subject to suppression. This behavior can be understood by expressing Eq. (74) in the form

$$
\delta_{c}^{\prime \prime}+a\left(H+\Gamma_{c}\right) \delta_{c}^{\prime}-4 \pi a^{2}\left(G_{c} \rho_{c} \delta_{c}+G \rho_{b} \delta_{b}\right) \simeq 0,
$$

where $G_{c}$ is the effective gravitational coupling for $\delta_{c}$ defined by

$$
G_{c} \equiv G-\frac{\dot{\Gamma}_{c}+2 H \Gamma_{c}}{4 \pi \rho_{c}} .
$$

Assuming that $|\alpha| \ll 1$ and expanding $G_{c}$ around $\alpha=0$, we obtain

$$
G_{c}=G\left[1+\alpha\left(1-\Omega_{m}\right) \frac{\tilde{\Omega}_{m}\left(3 \tilde{\Omega}_{m}+4\right)}{\tilde{\Omega}_{c}^{2}} \frac{H_{0}^{2}}{H^{2}}+O\left(\alpha^{2}\right)\right],
$$

where $\tilde{\Omega}_{m}=8 \pi G \rho_{m} /\left(3 H^{2}\right)$ and $\tilde{\Omega}_{c}=8 \pi G \rho_{c} /\left(3 H^{2}\right)$. For $\alpha>0$, we have $G_{c}>G$ and hence the growth of $\delta_{c}$ is enhanced in comparison to the $\Lambda$ CDM model. For $\Omega_{m}=0.32$ and $\Omega_{c}=0.27$, today's value of $G_{c}$ is given by $G_{c} \simeq G(1+14.8 \alpha)$. This means that, even for $|\alpha|=O(0.1), G_{c}$ is significantly modified relative to the $\alpha=0$ case. For $\alpha>0$, the term $a \Gamma_{c}$ in front of $\delta_{c}^{\prime}$ in Eq. 91 is negative, so this also works to enhance the growth rate of $\delta_{c}$. The enhancement of $\delta_{c}$ also leads to the larger baryon growth rate $f_{b}$ through Eq. (75) in comparison to the $\alpha=0$ case. As we see in the left panel of Fig. 1 , $f_{c}$ is larger than $f_{b}$ (see Ref. [106] for a related work). The evolution of total matter growth function is similar to that of CDM, but $f_{m}$ is slightly smaller than $f_{c}$ due to the presence of baryons.

For $\alpha<0$, the growth of CDM density contrast is suppressed in comparison to the $\alpha=0$ case. As we observe in the right panel of Fig. 11, the suppression of $f_{c}$ also gives rise to the values of $f_{b}$ and $f_{m}$ smaller than those in the $\Lambda$ CDM model. Thus, the large or small cosmic growth rates can be realized in the interacting vacuum energy scenario, depending on the sign of $\alpha$. This allows us to probe the signature of interactions observationally.

In RSD measurements, the matter velocity potential is used to quantify the galaxy distortion in redshift space. In Eq. (58) the term $-\dot{Q} \theta / \rho_{c}$ is neglected relative to the other terms for sub-horizon perturbations, so using the CDM growth rate $f_{c}$ leads to

$$
H\left(f_{c}+g_{c}\right) \delta_{c}=\frac{k^{2}}{a^{2}} \theta-\frac{1}{2} \dot{h}
$$

where $g_{c} \equiv Q /\left(H \rho_{c}\right)$. This means that the CDM growth rate associated with the velocity potential $\theta$ corresponds to [107, 108]

$$
f_{c, \mathrm{RSD}}=f_{c}+g_{c}
$$

Hence there is the additional contribution to $f_{c}$ from the coupling $Q$. From Eq. 65, the growth rate of baryon density contrast relevant to RSD measurements is simply given by $f_{b}$. As our tracers are luminous baryonic matter, we mostly use $\delta_{b}$ in our forecast analysis. However, the late-time enhancement or suppression in $\delta_{m}$ can lead to tight observational constraints on the interaction between vacuum energy and CDM, so we will also discuss the case of total matter density contrast.

\section{MATTER POWER SPECTRUM}

To confront the interacting vacuum energy model with the observations of luminous galaxies, we define the matter power spectrum in Fourier space at a redshift $z$, as

$$
\mathcal{P}_{L}(k, z)=\mathcal{P}_{L, 0}(k)\left(\frac{\delta_{b, m}(a)}{\delta_{b, m}(1)}\right)^{2},
$$

where we exploited the fact that Eq. (75) does not contain the $k$ dependence, and the suffices $b$ and $m$ refer to the baryonic and total matter scenarios which we consider separately in this work. The scale-dependent part $\mathcal{P}_{L, 0}(k)$ is today's matter power spectrum, which is given by

$$
\mathcal{P}_{L, 0}(k)=\mathcal{P}_{0} k^{n_{s}} \mathcal{T}^{2}(k),
$$

where the scaling constant $\mathcal{P}_{0}$ is found using the usual normalization of $\sigma_{8}$, i.e.,

$$
\sigma_{8,0}^{2}=\frac{1}{2 \pi^{2}} \int_{0}^{\infty} k^{2} W^{2}(k, R) \mathcal{P}_{L, 0}(k) \mathrm{d} k,
$$


with the window function

$$
W(k, R)=\frac{3}{k^{3} R^{3}}[\sin (k R)-k R \cos (k R)],
$$

at the comoving scale $R=8 h^{-1} \mathrm{Mpc}$. In Eq. 977, the scale dependence is present in the spectral index $n_{s}$ of primordial scalar perturbations and the transfer function $\mathcal{T}$. The latter accommodates the evolution of gravitational potentials from the radiation dominance to the matter era.

The galaxy linear power spectrum is defined as 11

$$
\mathcal{P}_{g}(k, z, \mu)=\left[b_{s}(z)+f_{s}(z) \mu^{2}\right]^{2} \frac{\mathcal{P}_{L, 0}(k)}{\sigma_{8,0}^{2}} \exp \left[-k^{2} \mu^{2} \Sigma_{z}^{2}(z)\right],
$$

where $b_{s}(z)=b_{g}(z) \sigma_{8}(z)$ and $f_{s}(z)=f_{b}(z) \sigma_{8}(z)$, with $b_{g}$ and $f_{b}$ being the galaxy bias and the baryonic growth rate, respectively. The cosine of the angle of unit wavevector $\boldsymbol{k}$ with respect to the line-of-sight direction is written as $\mu=\boldsymbol{k} \cdot \boldsymbol{r} / \boldsymbol{r}$. In Eq. $\left[100\right.$, we have inserted the damping factor $\exp \left[-k^{2} \mu^{2} \Sigma_{z}^{2}(z)\right]$ to account for redshift uncertainties $\sigma_{z}(z)$, weighed by $\Sigma_{z}(z)=\sigma_{z}(z) / H(z)$. Finally, the observed matter power spectrum can be written as

$$
\mathcal{P}_{\text {obs }}(k, z, \mu)=\mathcal{P}_{g}(k, z, \mu)+\mathcal{P}_{\text {shot }}(z),
$$

where the shot noise is defined by $\mathcal{P}_{\text {shot }}(z)=1 / n(z)$, with $n$ being the comoving galaxy density per redshift bin.

For the damping of non-linear evolution of the matter power spectrum, we follow the approach given in Refs. [109- 111]. On scales larger than $100 h^{-1} \mathrm{Mpc}$, i.e., $k \lesssim 0.06 h \mathrm{Mpc}^{-1}$, which is our range of interest, the non-linear evolution leads to a damping/suppression of all linear theory information, as shown in the left panel of Fig. 1 of Ref. [112] and discussed extensively in Refs. [109--114]. Such damping can be modelled by the non-linear power spectrum [109-111, 114]

$$
\mathcal{P}_{\mathrm{NL}}(k, z, \mu)=\mathcal{P}_{\mathrm{obs}}(k, z, \mu) \exp \left[-\frac{k^{2}}{2}\left(1-\mu^{2}\right) \Sigma_{\perp}^{2}(z)-\frac{k^{2}}{2} \mu^{2} \Sigma_{\|}^{2}(z)\right],
$$

where the damping factors are needed to take into account the smearing due to non-linear structure formation along $\left(\Sigma_{||}\right)$and across $\left(\Sigma_{\perp}\right)$ the line of sight [109, 110]. The damping factors are given by

$$
\begin{aligned}
& \Sigma_{\perp}(z)=0.785 \Sigma_{0} \frac{\delta_{b, m}(z)}{\delta_{b, m}(0)}, \\
& \Sigma_{||}(z)=\left[1+f_{b}(z)\right] \Sigma_{\perp}(z),
\end{aligned}
$$

where $\Sigma_{0}=11 h^{-1} \mathrm{Mpc}$. One should point out that the numerical factors which appear in the previous expressions are technically model dependent, i.e. for the interacting vacuum energy models they might be different from the case of a standard $\Lambda$ CDM. Although, it is generally assumed in the literature that their impact on the final Fisher analysis is mild, for they do not enter in the derivatives of the power spectrum with respect to the model parameters, thus effectively resulting in a sort of marginalization procedure [110].

\section{FORECASTS}

We exploit a Fisher Matrix to compute errors in the J-PAS forecast. In the case of a single tracer, a generic Fisher Matrix element, $F_{i j}$, can be calculated as [115]

$$
\begin{aligned}
F_{i j}(z) & =\frac{1}{4 \pi^{2}} \int_{-1}^{1} \mathrm{~d} \mu^{\prime} \int_{k_{\min }}^{k_{\max }} \mathrm{d} k^{\prime} k^{\prime 2} \frac{V_{\text {eff }}\left(k^{\prime}, z, \mu^{\prime}\right)}{2} \frac{\mathrm{d} \ln \mathcal{P}_{\text {obs }}\left(k^{\prime}, z, \mu^{\prime}\right)}{\mathrm{d} p_{i}} \frac{\mathrm{d} \ln \mathcal{P}_{\text {obs }}\left(k^{\prime}, z, \mu^{\prime}\right)}{\mathrm{d} p_{j}} \\
& \times \exp \left[-k^{\prime 2}\left(1-\mu^{\prime 2}\right) \Sigma_{\perp}^{2}(z)-k^{\prime 2} \mu^{\prime 2} \Sigma_{\|}^{2}(z)\right],
\end{aligned}
$$

where $k_{\min }=0.007 \mathrm{~h} \mathrm{Mpc}^{-1}$ and $k_{\max } \rightarrow \infty$ [116]. The effective volume of the survey is

$$
V_{\mathrm{eff}}(k, z, \mu)=V_{a}(z)\left[\frac{n(z) \mathcal{P}_{g}(k, z, \mu)}{n(z) \mathcal{P}_{g}(k, z, \mu)+1}\right]^{2},
$$

\footnotetext{
${ }^{1}$ As we showed in Eq. 95, the CDM power spectrum can be derived by performing the concomitant substitution $f_{b} \rightarrow f_{c}+g_{c}$ in Eq. 100 .
} 
Table I. Surveys specifications. Column 1: name of the survey; column 2: redshift range; column 3: redshift bin width; column 4: survey area in sq. deg.; column 5: redshift error; column 6: galaxy bias functions a

\begin{tabular}{cccccc}
\hline \hline & $\left(z_{\min }, z_{\max }\right)$ & $\Delta z$ & $\begin{array}{c}A_{\text {survey }} \\
\text { (sq. deg. })\end{array}$ & $\sigma_{z}$ & $b_{g}$ \\
\hline DESI-BCG & $(0.05,0.6)$ & & & $0.0005(1+z)$ & $1.34 \frac{\delta_{m, b}(0)}{\delta_{m, b}(z)}$ \\
DESI-LRG & $(0.6,1.2)$ & 0.2 & 14000 & $\frac{\delta_{m, b}(0)}{\delta_{m, b}(z)}$ \\
DESI-ELG & $(0.6,1.8)$ & & & $0.001(1+z)$ & $0.53+0.289(1+z)^{2}$ \\
DESI-QSO & $(0.6,1.8)$ & & & $0.001(1+z)$ & Table 3 in $[117$ \\
\hline \hline Euclid & $(0.9,1.8)$ & 0.2 & 15000 & & $1.7 \frac{\delta_{m, b}(0)}{\delta_{m, b}(z)}$ \\
\hline J-PAS-LRG & $(0.2,1.2)$ & & & $0.003(1+z)$ & $0.84 \frac{\delta_{m, b}(0)}{\delta_{m, b}(z)}$ \\
J-PAS-ELG & $(0.2,1.4)$ & 0.2 & $4000-8500$ & & $0.53+0.289(1+z)^{2}$ \\
J-PAS-QSO & $(0.2,4)$ & & & & \\
\hline \hline
\end{tabular}

a Note that the provided galaxy bias functions should be considered model dependent, thus for our interacting vacuum energy model they might be different. But the impact on the final Fisher analysis is smeared out by performing a marginalization over these functions, as described in the following pages.

where

$$
V_{a}(z)=\frac{4 \pi f_{\text {sky }}}{3}\left[\chi\left(\bar{z}_{a}\right)^{3}-\chi\left(\bar{z}_{a-1}\right)^{3}\right]
$$

is the volume span by the survey in each redshift bin, and

$$
\chi(z)=\int_{0}^{z} \frac{\mathrm{d} z^{\prime}}{H\left(z^{\prime}\right)}
$$

is the comoving distance with $\bar{z}_{a}$ being the upper limit of $a$-th redshift bin.

Since the surveys considered in our analysis will have more than one tracer with different biases used to probe the same patch of the sky at the same redshift range, we also take into account the cross correlation and define a generalized Fisher Matrix as [118, 119]

$$
\begin{aligned}
F_{i j}(z) & =\sum_{X, Y=1}^{N} \frac{1}{4 \pi^{2}} \int_{-1}^{1} \mathrm{~d} \mu^{\prime} \int_{k_{\min }}^{k_{\max }} \mathrm{d} k^{\prime} k^{\prime 2} V_{a}(z) \frac{\mathrm{d} \ln \widehat{P}_{X, \mathrm{obs}}\left(k^{\prime}, z, \mu^{\prime}\right)}{\mathrm{d} p_{i}} \widehat{F}_{X Y} \frac{\mathrm{d} \ln \widehat{P}_{Y, \mathrm{obs}}\left(k^{\prime}, z, \mu^{\prime}\right)}{\mathrm{d} p_{j}} \\
& \times \exp \left[-k^{\prime 2}\left(1-\mu^{\prime 2}\right) \Sigma_{\perp}^{2}(z)-k^{\prime 2} \mu^{\prime 2} \Sigma_{\|}^{2}(z)\right],
\end{aligned}
$$

where the indices $(X, Y)$ run over the galactic tracers, i.e., LRG, ELG, and QSO, and $N=2$ or 3 depending on the tracer (see Table If for survey specifications). Also, the dimensionless effective power $\widehat{P}_{X \text {,obs }}$ is defined as

$$
\widehat{P}_{X, \mathrm{obs}}=n_{X} \mathcal{P}_{X, \mathrm{obs}},
$$

where $n_{X}$ is the comoving galaxy density per redshift bin per galaxy population and the Fisher information density $\widehat{F}_{X Y}$ is written as

$$
\widehat{F}_{X Y}=\frac{1}{4}\left[\delta_{X Y} \frac{\widehat{P}_{X, \mathrm{obs}} \widehat{P}_{\mathrm{obs}}}{1+\widehat{P}_{\mathrm{obs}}}+\frac{\widehat{P}_{X, \mathrm{obs}} \widehat{P}_{Y, \mathrm{obs}}\left(1-\widehat{P}_{\mathrm{obs}}\right)}{\left(1+\widehat{P}_{\mathrm{obs}}\right)^{2}}\right]
$$

with $\widehat{P}_{\text {obs }}=\sum_{X} \widehat{P}_{X, \text { obs. }}$. Clearly, for the single-tracer case, Eq. 109 reduces to Eq. 105 .

Adopting the nomenclature of Ref. [120], we consider the following combinations in our analysis (also summarised in Table II]:

- “P $(k)$-marginalised-over-shape" Fisher Matrix, where only BAO information is taken into account (henceforth "BAO”); 
Table II. Fisher Matrix scenarios. Column 1: step in Fisher Matrix calculation; column 2: name of Fisher Matrix; column 3: parameters of the Fisher Matrix; column 4: comments.

\begin{tabular}{|c|c|c|c|}
\hline 0 & $\mathcal{F}^{\text {bin }}$ & $\begin{array}{c}\left\{\ln D_{A}, \ln H, f_{s}, b_{s}, \mathcal{P}_{\text {shot }}, \Omega_{m}, h, n_{s}\right\} \\
\left\{\ln D_{A}, \ln H, f_{s}, b_{s_{1}}, b_{s_{2}}, \mathcal{P}_{\text {shot }}, \Omega_{m}, h, n_{s}\right\} \\
\left\{\ln D_{A}, \ln H, f_{s}, b_{s_{1}}, b_{s_{2}}, b_{s_{3}}, \mathcal{P}_{\text {shot }}, \Omega_{m}, h, n_{s}\right\}\end{array}$ & $\begin{array}{l}\text { single tracer: } 8 \times 8 \text { matrix } \\
2 \text { tracers: } 9 \times 9 \text { matrix } \\
3 \text { tracers: } 10 \times 10 \text { matrix }\end{array}$ \\
\hline \multicolumn{4}{|c|}{$\mathrm{BAO}$} \\
\hline A1 & $\mathcal{F}_{\text {marg }}^{\text {bin }}$ & $\left\{\ln D_{A}, \ln H\right\}$ & $\begin{array}{l}\text { marginalization over }\left\{f_{s}, b_{s_{i}}, \mathcal{P}_{\text {shot }}, \Omega_{m}, h, n_{s}\right\} \\
2 \times 2 \text { matrix }\end{array}$ \\
\hline \multirow[t]{2}{*}{ A2 } & \multirow[t]{2}{*}{$\mathcal{F}_{\text {cosmo }}^{\text {bin }}$} & \multirow[t]{2}{*}{$\Omega_{m}, \alpha$} & $\begin{array}{l}\text { projection of } \mathcal{F}_{\text {marg }}^{\text {bin }} \text { onto cosmological parameters } \\
\qquad 2 \times 2 \text { matrix }\end{array}$ \\
\hline & & & redshift rows of Tables III IV $-\mathrm{V}-\mathrm{VI}$ \\
\hline A3 & $\mathcal{F}_{\text {cosmo }}^{\text {tot }}$ & $\left\{\Omega_{m}, \alpha\right\}$ & $\begin{array}{l}\text { sum of } \mathcal{F}_{\text {cosmo }}^{\text {bin }} \\
2 \times 2 \text { matrix } \\
\text { last row of Tables } \mathrm{III}-\mathrm{IV}-\mathrm{V}-\mathrm{VI}\end{array}$ \\
\hline \multicolumn{4}{|c|}{$\mathrm{BAO}+\mathrm{RSD}$} \\
\hline B1 & $\mathcal{F}_{\text {marg }}^{\text {bin }}$ & $\left\{\ln D_{A}, \ln H, f_{S}\right\}$ & $\begin{array}{c}\text { marginalization over }\left\{b_{s_{i}}, \mathcal{P}_{\text {shot }}, \Omega_{m}, h, n_{s}\right\} \\
3 \times 3 \text { matrix }\end{array}$ \\
\hline \multirow[t]{2}{*}{ B2 } & \multirow[t]{2}{*}{$\mathcal{F}_{\text {cosmo }}^{\text {bin }}$} & \multirow[t]{2}{*}{$\Omega_{m}, \alpha$} & $\begin{array}{l}\text { projection of } \mathcal{F}_{\text {marg }}^{\text {bin }} \text { onto cosmological parameters } \\
\qquad 2 \times 2 \text { matrix }\end{array}$ \\
\hline & & & redshift rows of Tables VII VIII $\mathrm{IX}-\mathrm{X}$ \\
\hline B3 & $\mathcal{F}_{\text {cosmo }}^{\text {tot }}$ & $\left\{\Omega_{m}, \alpha\right\}$ & 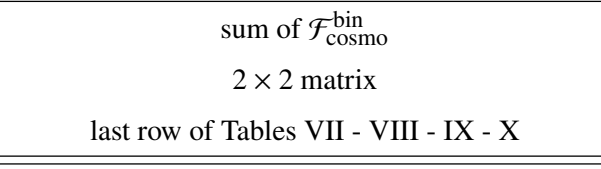 \\
\hline \multicolumn{4}{|c|}{$\mathrm{BAO}+\mathrm{RSD}+\mathrm{PS}$} \\
\hline $\mathrm{C} 1$ & $\mathcal{F}_{\text {marg }}^{\text {bin }}$ & $\left\{\ln D_{A}, \ln H, f_{s}, \Omega_{m}, h, n_{s}\right\}$ & $\begin{array}{l}\text { marginalization over }\left\{b_{s_{i}}, \mathcal{P}_{\text {shot }}\right\} \\
\qquad 6 \times 6 \text { matrix }\end{array}$ \\
\hline \multirow[t]{2}{*}{$\mathrm{C} 2$} & \multirow[t]{2}{*}{$\mathcal{F}_{\text {cosmo }}^{\text {bin }}$} & \multirow[t]{2}{*}{$\Omega_{m}, \alpha$} & $\begin{array}{l}\text { projection of } \mathcal{F}_{\text {marg }}^{\text {bin }} \text { onto cosmological parameters } \\
\qquad 2 \times 2 \text { matrix }\end{array}$ \\
\hline & & & redshift rows of Tables XI-XII-XIII-XIV \\
\hline \multirow[t]{2}{*}{$\mathrm{C} 3$} & \multirow[t]{2}{*}{$\mathcal{F}_{\text {cosmo }}^{\text {tot }}$} & \multirow[t]{2}{*}{$\Omega_{m}, \alpha$} & $\begin{array}{l}\text { sum of } \mathcal{F}_{\text {cosmo }}^{\text {bin }} \\
2 \times 2 \text { matrix }\end{array}$ \\
\hline & & & last row of Tables XI-XII $-\mathrm{XIII}-\mathrm{XIV}$ \\
\hline
\end{tabular}

- “P $(k)$-marginalised-over-shape" Fisher Matrix, with both BAO and RSD included (henceforth "BAO+RSD”);

- "full $\mathcal{P}(k)$ method with growth information included" Fisher Matrix, where the power spectrum broadband "shapeparameters" are added to BAO and RSD (henceforth "BAO+RSD+PS").

In order to avoid numerical instabilities, we follow the procedure reported in Ref. [121] for marginalisation $]$,

In Table II we define: the general Fisher matrix in each redshift bin, $\mathcal{F}^{\text {bin }}$; its marginalization over the uninformative parameter $\sqrt{3}$ depending on the considered scenario, $\mathcal{F}_{\text {marg }}^{\text {bin }}$; the projection of $\mathcal{F}_{\text {marg }}^{\text {bin }}$ onto the relevant two-dimensional parameter space related to the interacting model $\left(\mathcal{F}_{\text {cosmo }}^{\text {bin }}\right)$ in each redshift bin; and $\mathcal{F}_{\text {cosmo }}^{\text {tot }}$, the total Fisher onto these two parameters obtained

\footnotetext{
${ }^{2}$ Note that the shot noise $P_{\text {shot }}$ makes the matrix ill-conditioned. After applying the check as in Eq. (14) of Ref. [121], we have effectively verified that the corresponding eigenvalue is much smaller than those related to the other parameters, and that the corresponding row is near zero. Thus, the shot noise component is uncorrelated with the parameters we are interested in, and we can safely ignore its contribution by cutting the corresponding rows and columns from the starting Fisher matrix.

${ }^{3}$ Note that the marginalization over the bias
} 

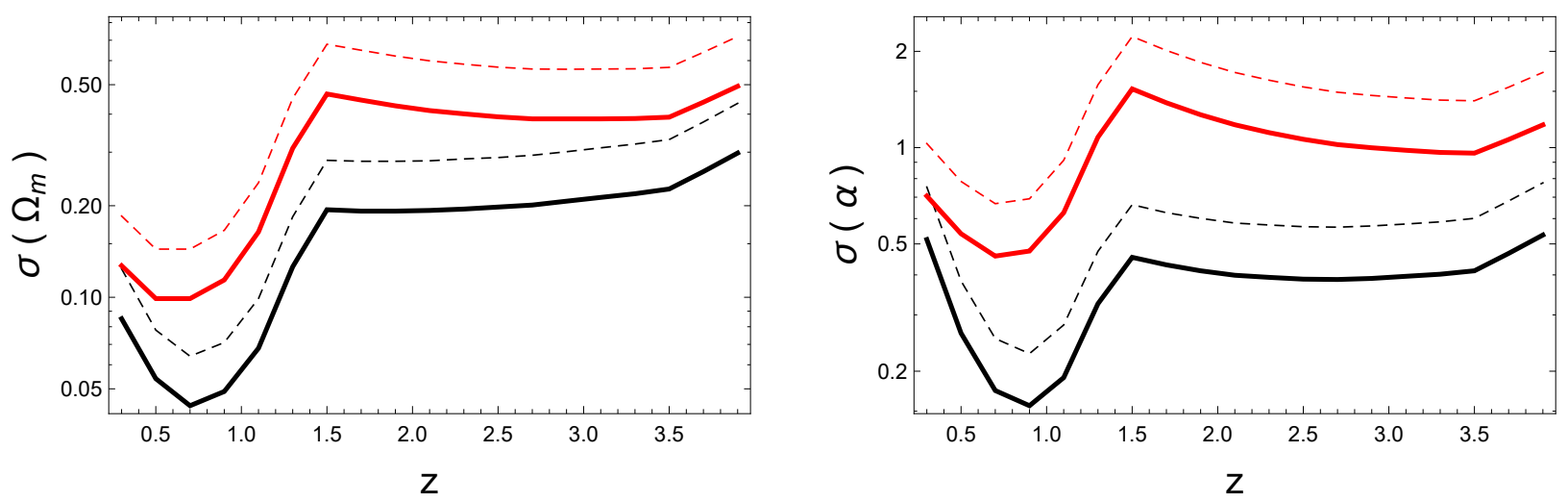

Figure 2. Comparison of the J-PAS estimated errors for the interaction parameter $\alpha$ and the matter density parameter $\Omega_{m}$ with the BAO+RSD data. The red and black lines correspond to the case in which the baryonic and total matter growing functions are used in the analysis, respectively. Thin and thick lines are the plots for J-PAS $4000 \mathrm{deg}^{2}$ and $8500 \mathrm{deg}^{2}$, respectively.

summing up contribution from each redshift bin. The values reported in our Tables are derived from $\mathcal{F}_{\text {cosmo }}^{\text {bin }}$ and $\mathcal{F}_{\text {cosmo }}^{\text {tot }}$ after inversion, i.e., the errors on a given parameter are obtained from $\sigma_{p_{i}}=\sqrt{F_{i i}^{-1}}$.

In this analysis, we generate the transfer function with the Boltzmann code CLASS [122] adapted to our perturbation equations. Our fiducial cosmology is a flat $\Lambda$ CDM model with the following parameters: $\Omega_{m}=0.31, h=0.68, n_{s}=0.96$, and $\sigma_{8,0}=0.82$. As mentioned earlier, the surveys considered in this analysis are J-PAS [79, 80], DESI [76], and Euclid [77]. The surveys specifications used in our forecast analysis can be found in Table $\mathbb{I}$ and also in Refs. [117, 123]. For completeness, all the quantities used to obtain the Fisher matrices elements are shown in the Appendix A.

\section{RESULTS}

In this section we summarize the main results of the present work. In Figs. 2 7 , we plot the J-PAS forecasted errors on $\Omega_{m}$ and $\alpha$ as functions of the redshift. In Appendixes B, C, D, we present the errors at different redshifts derived from the analysis of $\mathrm{BAO}, \mathrm{BAO}+\mathrm{RSD}$, and BAO+RSD+PS, respectively. In Tables III-XIV, we also show the errors found when we use $\delta_{m}$ instead of $\delta_{b}$ in Eq. (100) for the sake of completeness and comparison, together with those for DESI and Euclid.

In Figs. 2 and 3, the errors on the parameters $\Omega_{m}$ and $\alpha$ are larger when only baryonic tracers are considered in most of the redshift range. This is attributed to the fact that $\delta_{m}$ is subject to stronger enhancement or suppression in comparison to $\delta_{b}$ (see Fig. 17, so the analysis based on the total matter contrast gives rise to tighter bounds on the coupling $\alpha$. In Fig. 4 the difference between baryonic and total matter is particularly clear for ELGs and LRGs at low redshifts, where BAO and RSD are used with single tracers. As we observe in Fig. 5. constraining the whole power spectrum leads to more accurate results as compared to the case where only BAO and RSD are fitted.

In Figs. 6 and 7, we plot the estimated errors for $\Omega_{m}$ and $\alpha$ derived by using multi-tracers for both total matter and baryons. Besides J-PAS, we also show estimations of the DESI and Euclid surveys for comparison. We see from our results that J-PAS is competitive to DESI and Euclid for $z<0.6$, which confirms the results of a previous J-PAS forecast on non-interacting dark energy models and modified gravity theories [123].

Finally, we show in Figs. 8 and 9 the confidence regions in the $\left\{\Omega_{m}, \alpha\right\}$ plane for BAO+RSD and BAO+RSD+PS, respectively. We see that J-PAS with $8500 \mathrm{deg}^{2}$ is very competitive to the other two surveys considered in our analysis. At low redshifts $(z<0.6)$, when the presence of dark energy and the dark sector interaction is more relevant, J-PAS provides the best constraints on the parameters thanks to its high galaxy densities detectable in that redshift range. In the BAO+RSD+PS case, the J-PAS predicted errors are comparable to the actual errors derived from JLA SN Ia + Planck CMB data [21]. The full tables, showing all the results derived in our analysis, are shown in Appendices B, C and D.

\section{CONCLUSIONS}

The goal of the analysis performed in this paper is to report the J-PAS forecasts for the parameter estimation in a class of interacting vacuum energy models. The vacuum energy does not give rise to an additional dynamical degree of freedom, but the 

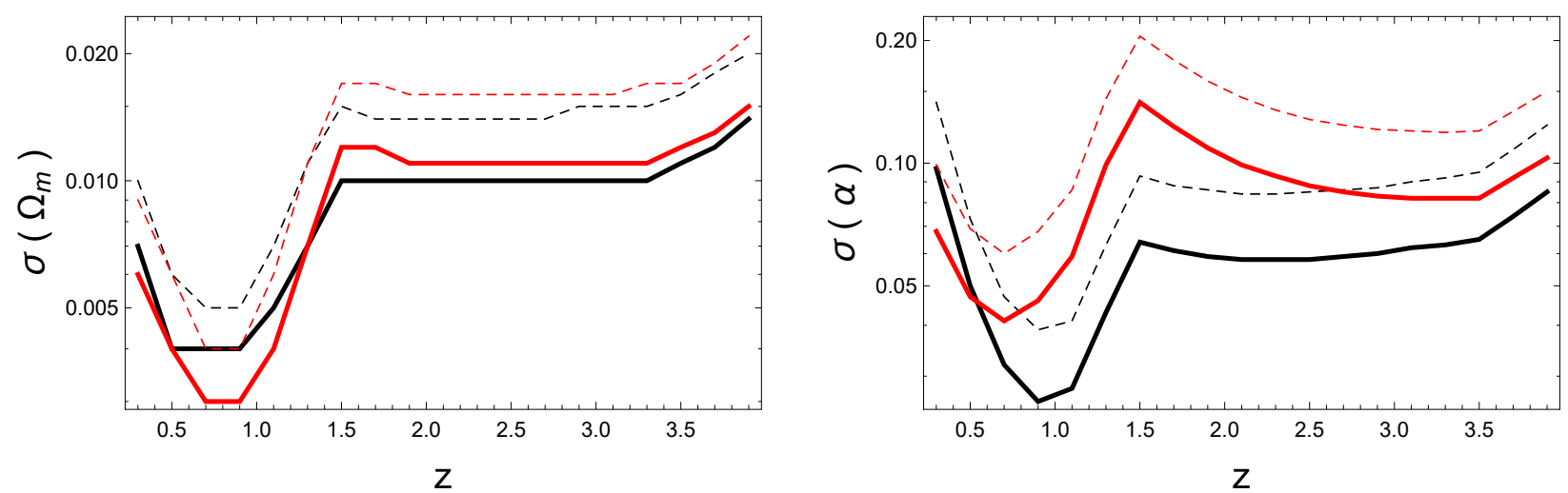

Figure 3. The same as Fig. 2, but with the analysis based on BAO + RSD + PS.
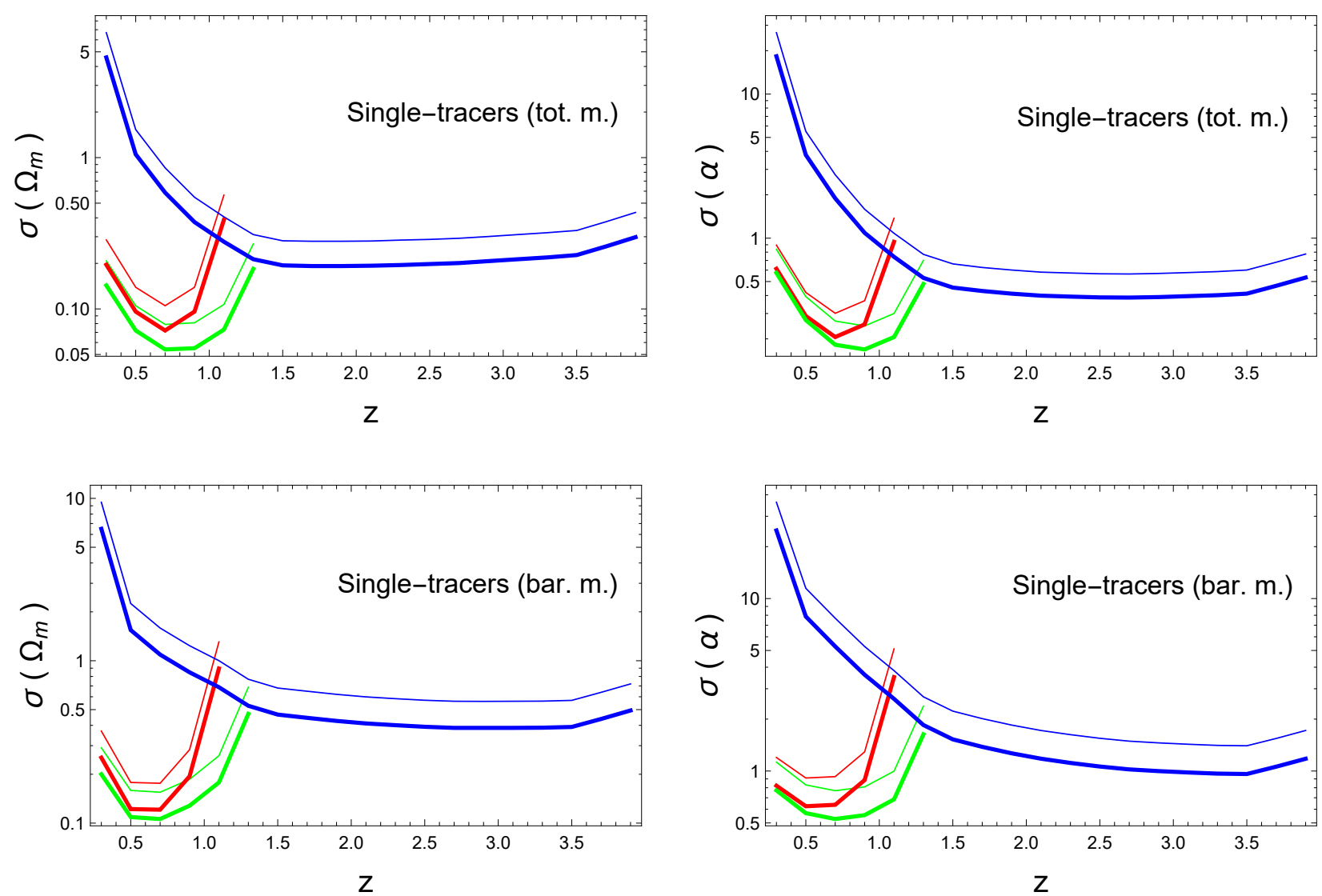

Figure 4. Estimated error for $\Omega_{m}$ and $\alpha$ as a function of $z$ with the BAO+RSD data. The estimations are from ELGs (green), LRGs (red) and QSOs (blue) power spectra for J-PAS survey in both $4000 \mathrm{deg}^{2}$ (thin lines) and $8500 \mathrm{deg}^{2}$ (thick lines) regions.

interaction between vacuum energy and CDM modifies the background cosmological dynamics through an energy exchange. At the level of perturbations, there are in general contributions to the interacting four vector $Q^{\mu}$ arising from energy and momentum transfers, which are weighed by $Q \mu^{\mu}$ and $q^{\mu}$ respectively. We focused on the case where the momentum transfer is absent $\left(q^{\mu}=0\right)$, under which the four velocities of vacuum energy and CDM are equivalent to each other. Then the vacuum energy perturbation $\delta \Lambda$ is related to the CDM four velocity potential $\theta$ as $\delta \Lambda=Q \theta$, so the system of perturbation equations of motion is closed. 

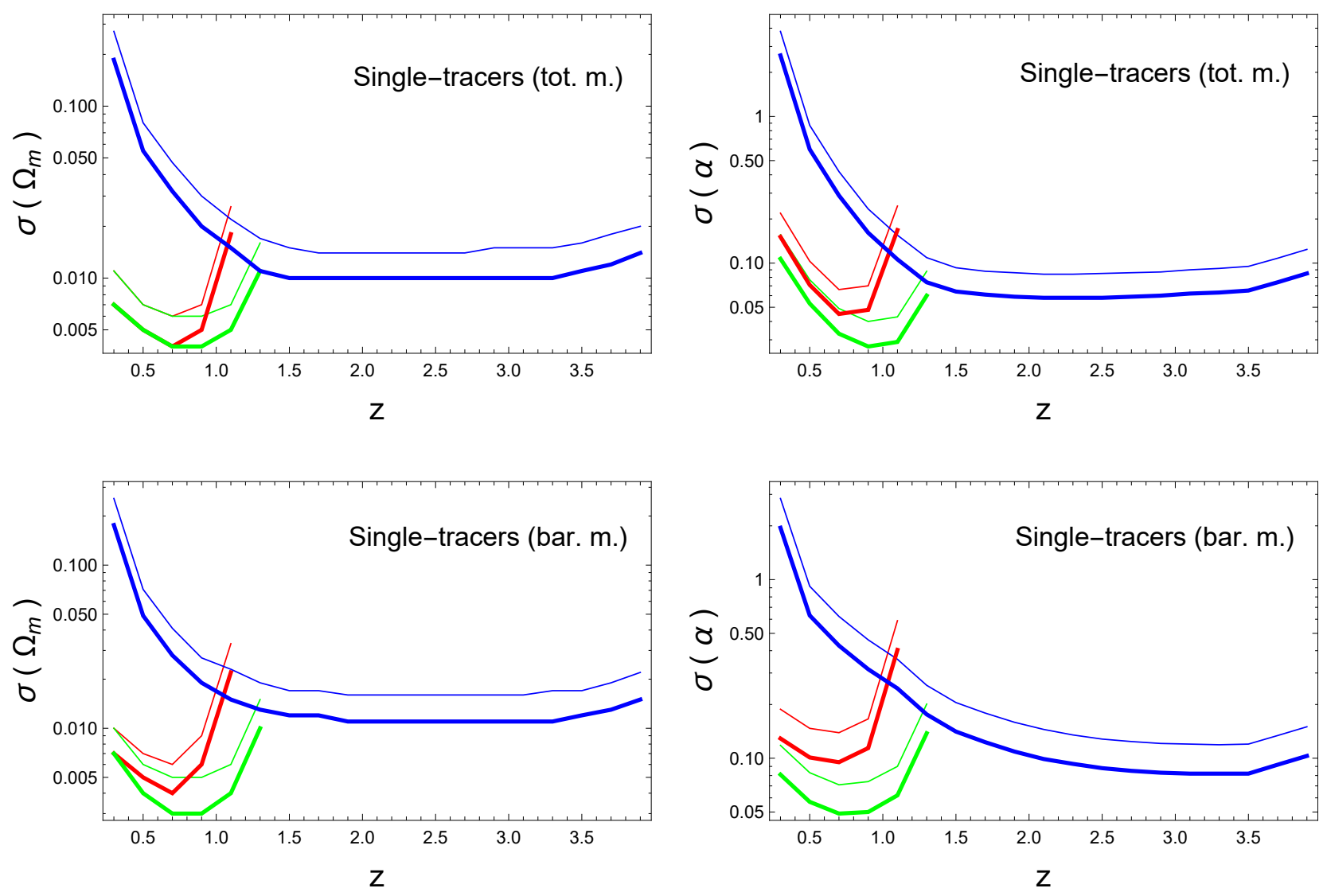

Figure 5. The same as Fig. 4, but with the analysis based on BAO + RSD + PS.

The interacting vacuum energy is chosen to be of the form 81 with $\sigma$ given by 82 , in which case $Q=-\alpha \sigma H^{-2 \alpha-1} \rho_{m}$. Then, the Hubble parameter is analytically known as a function of the redshift, see Eq. (84). The coupling constant $\alpha$ is the only additional parameter to those appearing in the $\Lambda \mathrm{CDM}$ model $(\alpha=0)$. For $\alpha>0$, the growth rate of CDM density contrast $\delta_{c}$ is enhanced in comparison to the $\Lambda \mathrm{CDM}$. This also leads to the larger growth rates of baryon and total matter density contrasts. For $\alpha<0$, the evolution of $\delta_{c}, \delta_{b}$, and $\delta_{m}$ is suppressed relative to the $\alpha=0$ case. These properties manifest themselves in the observations of the galaxy power spectrum including BAO and RSD.

We carried out the J-PAS forecasts for the matter density parameter $\Omega_{m}$ and the coupling $\alpha$. J-PAS will cover a region of 8500 $\mathrm{deg}^{2}$ in the northern sky. We extended the analysis for a smaller covering of $4000 \mathrm{deg}^{2}$ with a more conservative estimation of the impact of the data. For a $8500 \mathrm{deg}^{2}$ covering, it has been shown that J-PAS will lead to parameter estimations comparable to those obtained from DESI and Euclid for the same class of interacting models. In particular, for the redshift range $z<0.6$, the error bars in J-PAS measurements can be even smaller than those provided by DESI and Euclid - see Figs. 6and7 7. In general, the J-PAS constraints will tighten considerably the estimations of those parameters with respect to previous large-scale structure data, with precision comparable to that we have with actual CMB data. We conclude that confirming or ruling out the $\Lambda \mathrm{CDM}$ model by finding an interaction signature in a joint analysis of SN Ia, CMB, and large-scale structure data, with any of the three surveys considered in our analysis or by combining them, is a real and promising possibility.

\section{ACKNOWLEDGEMENTS}

We are thankful to our colleagues of J-PAS Theory Working Group for helpful discussions. MB acknowledges Istituto Nazionale di Fisica Nucleare (INFN), sezione di Napoli, iniziativa specifica QGSKY. RvM acknowledges support from the Programa de Capacitação Institucional do Observatorio Nacional PCI/ON/MCTI. SC is supported by CNPq (Grants No. 307467/2017-1 and 420641/2018-1). JA is supported by CNPq (Grants No. 310790/2014-0 and 400471/2014-0) and FAPERJ (Grant No. 233906). JCF is supported by CNPq (Grant No. 304521/2015-9) and FAPES (Grant No. 78/2017). ST is supported by the Grant-in-Aid 

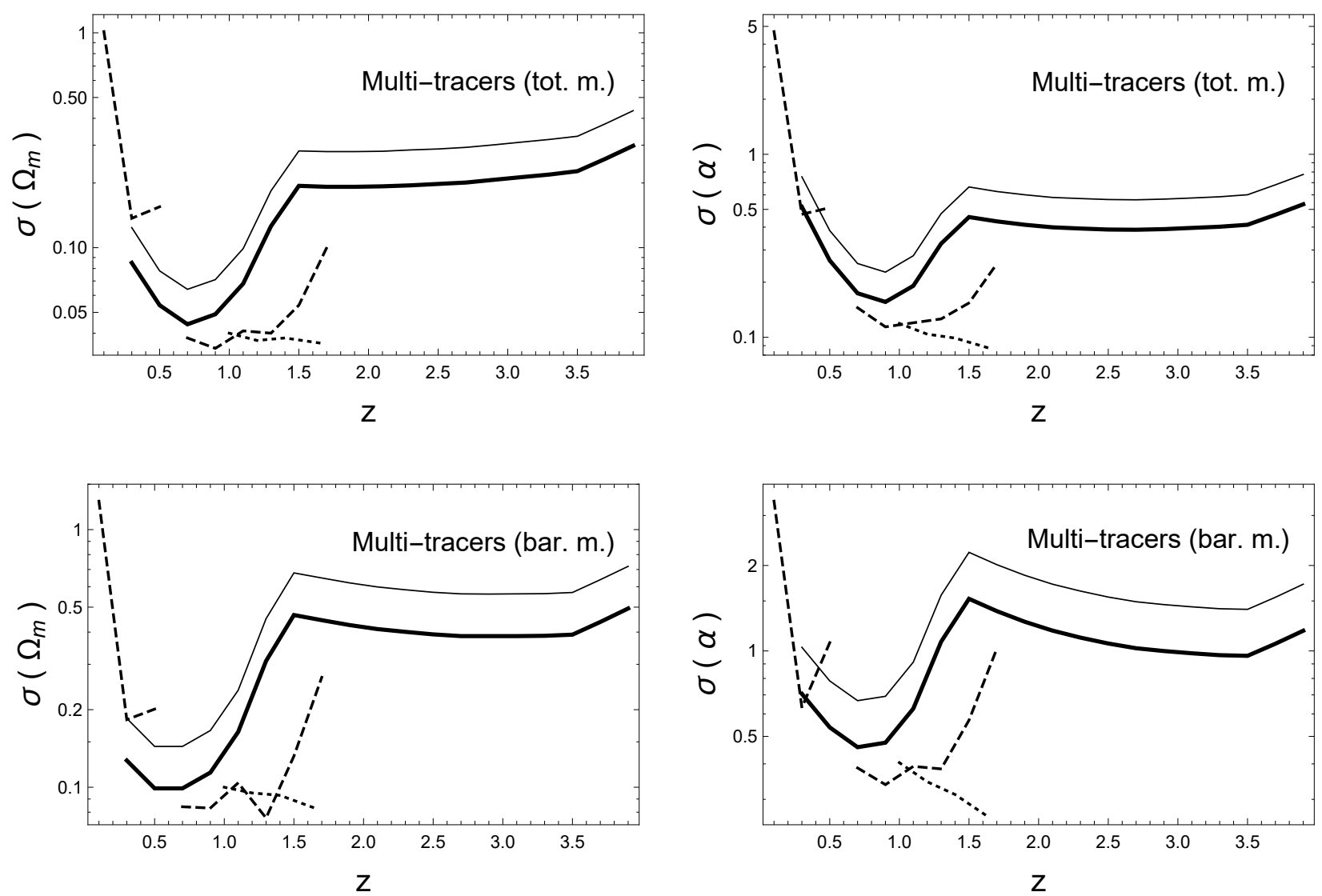

Figure 6. Estimated error for $\Omega_{m}$ and $\alpha$ as a function of $z$ with the BAO+RSD data. We show estimations from multi-tracers for Euclid (dotted), DESI (dashed; BGS at lower redshifts; LRG+ELG+QSO at higher redshifts), and for J-PAS survey in both 4000 deg $^{2}$ (thin solid) and 8500 $\operatorname{deg}^{2}$ (thick solid) regions.

for Scientific Research Fund of the JSPS No. 19K03854. VM thanks CNPq (Brazil) and FAPES (Brazil) for partial financial support. This project has received funding from the European Union's Horizon 2020 research and innovation programme under the Marie Skłodowska-Curie grant agreement No 888258.

This paper has gone through internal review by the J-PAS collaboration. Funding for the J-PAS Project has been provided by the Governments of Spain and Aragón through the Fondo de Inversión de Teruel, European FEDER funding and the MINECO and by the Brazilian agencies FINEP, FAPESP, FAPERJ and by the National Observatory of Brazil.

[1] E. J. Copeland, M. Sami, and S. Tsujikawa, Int. J. Mod. Phys. D 15, 1753 (2006), arXiv:hep-th/0603057

[2] T. Clifton, P. G. Ferreira, A. Padilla, and C. Skordis, Phys. Rept. 513, 1 (2012) arXiv:1106.2476 [astro-ph.CO]

[3] A. Joyce, B. Jain, J. Khoury, and M. Trodden, Phys. Rept. 568, 1 (2015) arXiv:1407.0059 [astro-ph.CO]

[4] R. Kase and S. Tsujikawa, Int. J. Mod. Phys. D 28, 1942005 (2019), arXiv:1809.08735 [gr-qc]

[5] P. J. E. Peebles, Astrophys. J. 284, 439 (1984)

[6] P. J. E. Peebles, Astrophys. J. Lett. 263, L1 (1982).

[7] N. Aghanim et al. (Planck), Astron. Astrophys. 641, A6 (2020) arXiv:1807.06209 [astro-ph.CO]

[8] A. G. Riess, S. Casertano, W. Yuan, L. M. Macri, and D. Scolnic, Astrophys. J. 876, 85 (2019), arXiv:1903.07603 [astro-ph.CO]

[9] W. L. Freedman, B. F. Madore, D. Hatt, T. J. Hoyt, I. S. Jang, R. L. Beaton, C. R. Burns, M. G. Lee, A. J. Monson, J. R. Neeley, M. M. Phillips, J. A. Rich, and M. Seibert, Astrophys. J. 882, 34 (2019) arXiv:1907.05922 [astro-ph.CO]

[10] M. Ozer and M. O. Taha, Nucl. Phys. B 287, 776 (1987)

[11] K. Freese, F. C. Adams, J. A. Frieman, and E. Mottola, Nucl. Phys. B 287, 797 (1987)

[12] M. S. Berman, Phys. Rev. D 43, 1075 (1991)

[13] D. Pavon, Phys. Rev. D 43, 375 (1991). 

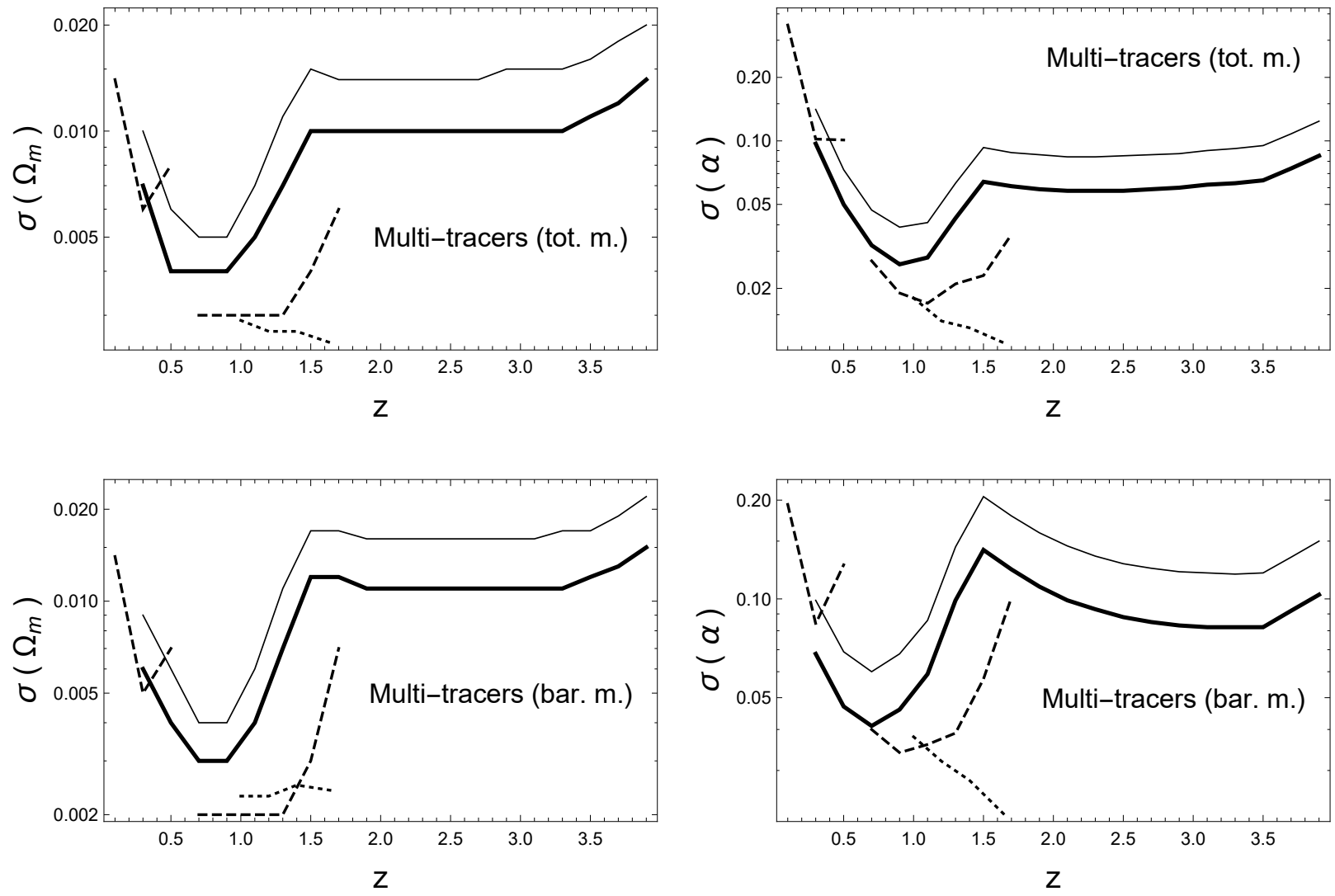

Figure 7. The same as Fig. 6, but with the analysis based on BAO + RSD + PS.
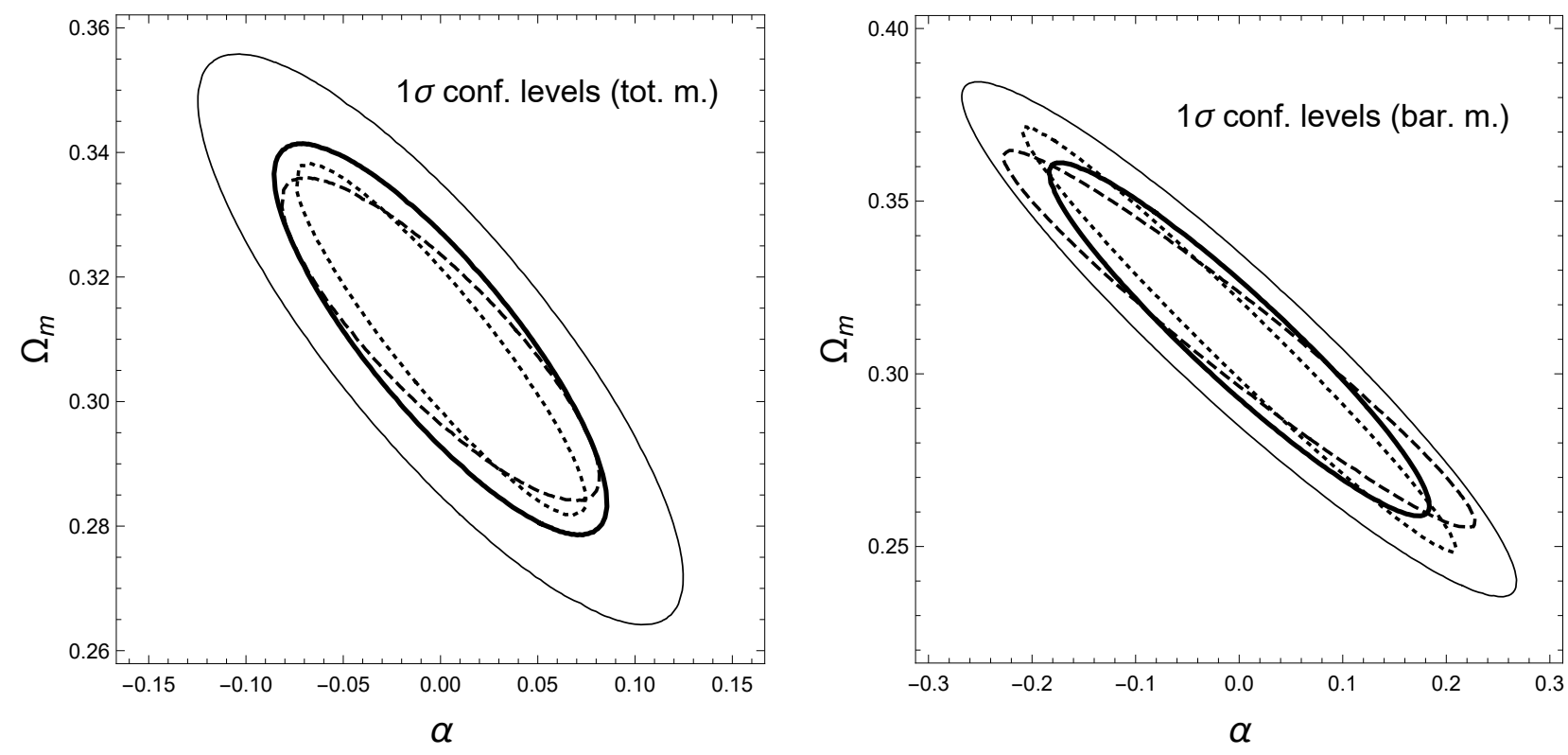

Figure 8. $1 \sigma$ contour error around $\alpha=0$ and $\Omega_{m}=0.31$ using multi-tracers for J-PAS $4000 \mathrm{deg}^{2}$ (thin solid), J-PAS 8500 deg${ }^{2}$ (thick solid), DESI (dashed) and Euclid (dotted) surveys, with the BAO+RSD data. 

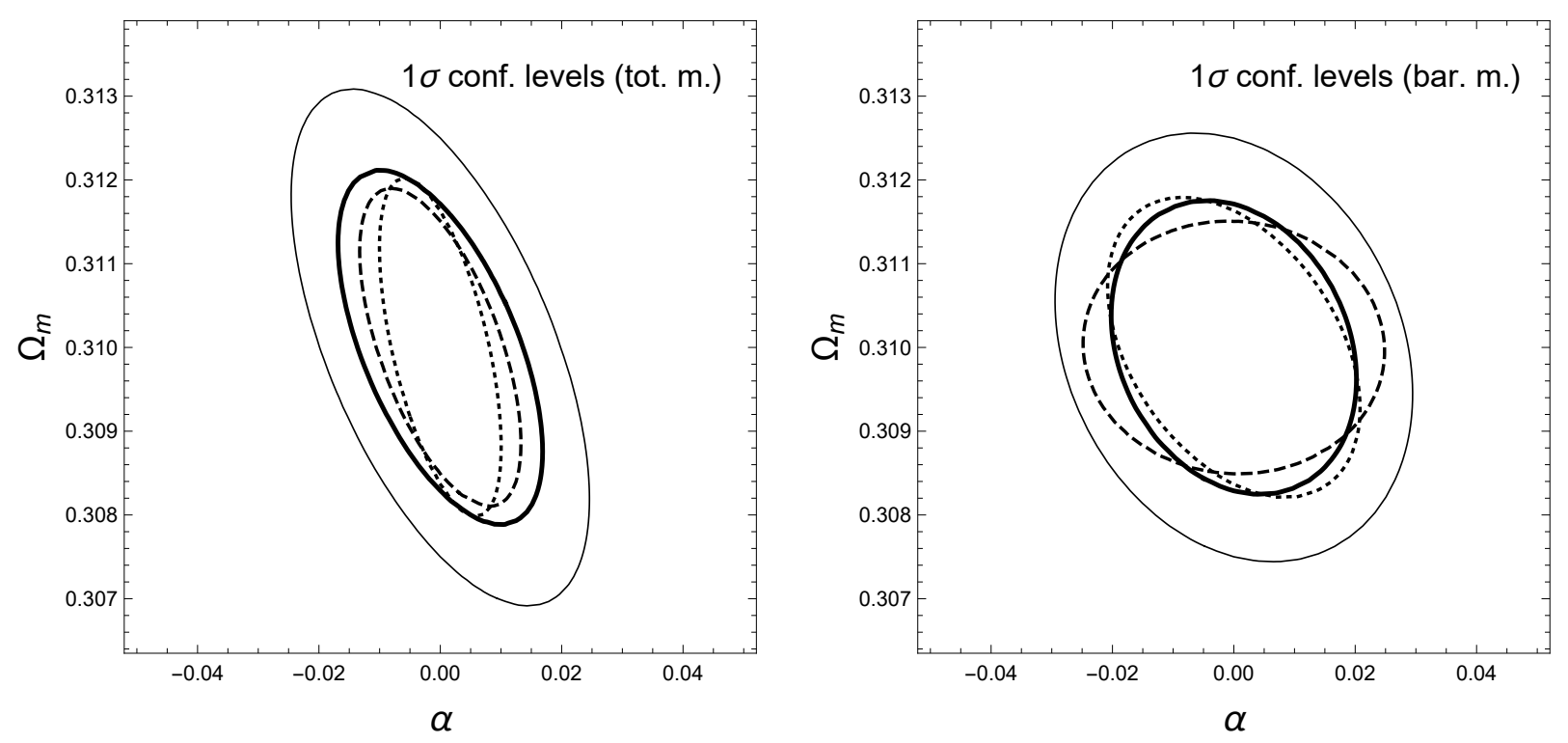

Figure 9. The same as Fig. 8, but with the analysis based on BAO + RSD + PS.

[14] J. C. Carvalho, J. A. S. Lima, and I. Waga, Phys. Rev. D 46, 2404 (1992)

[15] I. L. Shapiro and J. Sola, JHEP 02, 006 (2002), arXiv:hep-th/0012227

[16] J. S. Alcaniz, H. A. Borges, S. Carneiro, J. C. Fabris, C. Pigozzo, and W. Zimdahl, Phys. Lett. B 716, 165 (2012), arXiv:1201.5919 [astro-ph.CO]

[17] N. C. Devi, H. A. Borges, S. Carneiro, and J. S. Alcaniz, Mon. Not. Roy. Astron. Soc. 448, 37 (2015), arXiv:1407.1821 [astro-ph.CO]

[18] W. Zimdahl, H. A. Borges, S. Carneiro, J. C. Fabris, and W. S. Hipolito-Ricaldi, JCAP 04, 028 (2011) arXiv:1009.0672 [astro-ph.CO]

[19] D. Wands, J. De-Santiago, and Y. Wang, Class. Quant. Grav. 29, 145017 (2012), arXiv:1203.6776 [astro-ph.CO]

[20] V. Salvatelli, N. Said, M. Bruni, A. Melchiorri, and D. Wands, Phys. Rev. Lett. 113, 181301 (2014) arXiv:1406.7297 [astro-ph.CO]

[21] M. Benetti, W. Miranda, H. A. Borges, C. Pigozzo, S. Carneiro, and J. S. Alcaniz, JCAP 12, 023 (2019) arXiv:1908.07213 [astro-ph.CO]

[22] R. von Marttens, L. Casarini, D. F. Mota, and W. Zimdahl, Phys. Dark Univ. 23, 100248 (2019) arXiv:1807.11380 [astro-ph.CO]

[23] L. Xu, Y. Wang, M. Tong, and H. Noh, Phys. Rev. D 84, 123004 (2011) arXiv:1112.5216 [astro-ph.CO].

[24] K. Koyama, R. Maartens, and Y.-S. Song, JCAP 10, 017 (2009) arXiv:0907.2126 [astro-ph.CO]

[25] N. Dalal, K. Abazajian, E. E. Jenkins, and A. V. Manohar, Phys. Rev. Lett. 87, 141302 (2001) arXiv:astro-ph/0105317

[26] W. Zimdahl, D. Pavon, and L. P. Chimento, Phys. Lett. B 521, 133 (2001) arXiv:astro-ph/0105479.

[27] W. Zimdahl, D. J. Schwarz, A. B. Balakin, and D. Pavon, Phys. Rev. D 64, 063501 (2001) arXiv:astro-ph/0009353.

[28] L. P. Chimento, A. S. Jakubi, D. Pavon, and W. Zimdahl, Phys. Rev. D 67, 083513 (2003) arXiv:astro-ph/0303145.

[29] G. R. Farrar and P. J. E. Peebles, Astrophys. J. 604, 1 (2004) arXiv:astro-ph/0307316

[30] H. A. Borges and S. Carneiro, Gen. Rel. Grav. 37, 1385 (2005) arXiv:gr-qc/0503037

[31] J. S. Alcaniz and J. A. S. Lima, Phys. Rev. D 72, 063516 (2005) arXiv:astro-ph/0507372

[32] L. Amendola, G. Camargo Campos, and R. Rosenfeld, Phys. Rev. D 75, 083506 (2007) arXiv:astro-ph/0610806.

[33] H. Wei and S. N. Zhang, Phys. Lett. B 644, 7 (2007) arXiv:astro-ph/0609597

[34] M. R. Setare, Phys. Lett. B 642, 1 (2006), arXiv:hep-th/0609069

[35] H. M. Sadjadi and M. Alimohammadi, Phys. Rev. D 74, 103007 (2006) arXiv:gr-qc/0610080

[36] S. Das, P. S. Corasaniti, and J. Khoury, Phys. Rev. D 73, 083509 (2006) arXiv:astro-ph/0510628

[37] Z.-K. Guo, N. Ohta, and S. Tsujikawa, Phys. Rev. D 76, 023508 (2007) arXiv:astro-ph/0702015

[38] O. Bertolami, F. Gil Pedro, and M. Le Delliou, Phys. Lett. B 654, 165 (2007) arXiv:astro-ph/0703462.

[39] J. Valiviita, E. Majerotto, and R. Maartens, JCAP 07, 020 (2008) arXiv:0804.0232 [astro-ph].

[40] M. B. Gavela, D. Hernandez, L. Lopez Honorez, O. Mena, and S. Rigolin, JCAP 07, 034 (2009), [Erratum: JCAP 05, E01 (2010)], arXiv:0901.1611 [astro-ph.CO]

[41] S. del Campo, R. Herrera, and D. Pavon, JCAP 01, 020 (2009) arXiv:0812.2210 [gr-qc]

[42] I. L. Shapiro and J. Sola, Phys. Lett. B 682, 105 (2009) arXiv:0910.4925 [hep-th]

[43] L. P. Chimento, Phys. Rev. D 81, 043525 (2010) arXiv:0911.5687 [astro-ph.CO]

[44] T. Clemson, K. Koyama, G.-B. Zhao, R. Maartens, and J. Valiviita, Phys. Rev. D 85, 043007 (2012) arXiv:1109.6234 [astro-ph.CO]

[45] B. Wang, E. Abdalla, F. Atrio-Barandela, and D. Pavon, Rept. Prog. Phys. 79, 096901 (2016) arXiv:1603.08299 [astro-ph.CO]

[46] A. Cid, B. Santos, C. Pigozzo, T. Ferreira, and J. Alcaniz, JCAP 03, 030 (2019) arXiv:1805.02107 [astro-ph.CO] 
[47] S. Kumar and R. C. Nunes, Phys. Rev. D 94, 123511 (2016) arXiv:1608.02454 [astro-ph.CO]

[48] R. Murgia, S. Gariazzo, and N. Fornengo, JCAP 04, 014 (2016) arXiv:1602.01765 [astro-ph.CO].

[49] E. Di Valentino, A. Melchiorri, and O. Mena, Phys. Rev. D 96, 043503 (2017) arXiv:1704.08342 [astro-ph.CO].

[50] R. An, C. Feng, and B. Wang, JCAP 02, 038 (2018) arXiv:1711.06799 [astro-ph.CO]

[51] W. Yang, S. Pan, E. Di Valentino, R. C. Nunes, S. Vagnozzi, and D. F. Mota, JCAP 09, 019 (2018) arXiv:1805.08252 [astro-ph.CO]

[52] S. Pan, W. Yang, E. Di Valentino, E. N. Saridakis, and S. Chakraborty, Phys. Rev. D 100, 103520 (2019) arXiv:1907.07540 [astro-ph.CO]

[53] E. Di Valentino, A. Melchiorri, O. Mena, and S. Vagnozzi, Phys. Dark Univ. 30, 100666 (2020) arXiv:1908.04281 [astro-ph.CO]

[54] W. Yang, S. Pan, R. C. Nunes, and D. F. Mota, JCAP 04, 008 (2020) arXiv:1910.08821 [astro-ph.CO].

[55] E. Di Valentino, A. Melchiorri, O. Mena, and S. Vagnozzi, Phys. Rev. D 101, 063502 (2020) arXiv:1910.09853 [astro-ph.CO]

[56] S. Vagnozzi, L. Visinelli, O. Mena, and D. F. Mota, Mon. Not. Roy. Astron. Soc. 493, 1139 (2020). arXiv:1911.12374 [gr-qc]

[57] C. Wetterich, Astron. Astrophys. 301, 321 (1995), arXiv:hep-th/9408025.

[58] L. Amendola, Phys. Rev. D 62, 043511 (2000), arXiv:astro-ph/9908023

[59] A. Pourtsidou, C. Skordis, and E. J. Copeland, Phys. Rev. D 88, 083505 (2013) arXiv:1307.0458 [astro-ph.CO]

[60] C. G. Boehmer, N. Tamanini, and M. Wright, Phys. Rev. D 91, 123002 (2015) arXiv:1501.06540 [gr-qc]

[61] C. G. Boehmer, N. Tamanini, and M. Wright, Phys. Rev. D 91, 123003 (2015) arXiv:1502.04030 [gr-qc]

[62] C. Skordis, A. Pourtsidou, and E. J. Copeland, Phys. Rev. D 91, 083537 (2015) arXiv:1502.07297 [astro-ph.CO]

[63] T. S. Koivisto, E. N. Saridakis, and N. Tamanini, JCAP 09, 047 (2015) arXiv:1505.07556 [astro-ph.CO]

[64] A. Pourtsidou and T. Tram, Phys. Rev. D 94, 043518 (2016), arXiv:1604.04222 [astro-ph.CO]

[65] J. Dutta, W. Khyllep, and N. Tamanini, Phys. Rev. D 95, 023515 (2017), arXiv:1701.00744 [gr-qc]

[66] M. S. Linton, A. Pourtsidou, R. Crittenden, and R. Maartens, JCAP 04, 043 (2018) arXiv:1711.05196 [astro-ph.CO]

[67] R. Kase and S. Tsujikawa, Phys. Rev. D 101, 063511 (2020), arXiv:1910.02699 [gr-qc]

[68] R. Kase and S. Tsujikawa, Phys. Lett. B 804, 135400 (2020), arXiv:1911.02179 [gr-qc]

[69] F. N. Chamings, A. Avgoustidis, E. J. Copeland, A. M. Green, and A. Pourtsidou, Phys. Rev. D 101, 043531 (2020) arXiv:1912.09858 [astro-ph.CO]

[70] L. Amendola and S. Tsujikawa, JCAP 06, 020 (2020) arXiv:2003.02686 [gr-qc].

[71] R. Kase and S. Tsujikawa, JCAP 11, 032 (2020), arXiv:2005.13809 [gr-qc]

[72] A. De Felice, S. Nakamura, and S. Tsujikawa, Phys. Rev. D 102, 063531 (2020), arXiv:2004.09384 [gr-qc]

[73] M. Asghari, J. Beltrán Jiménez, S. Khosravi, and D. F. Mota, JCAP 04, 042 (2019) arXiv:1902.05532 [astro-ph.CO]

[74] J. B. Jiménez, D. Bettoni, D. Figueruelo, and F. A. Teppa Pannia, JCAP 08, 020 (2020) arXiv:2004.14661 [astro-ph.CO]

[75] R. Figuerelo and et al., in preparation (2021).

[76] A. Aghamousa et al. (DESI), (2016), arXiv:1611.00036 [astro-ph.IM]

[77] R. Laureijs et al. (EUCLID), (2011), arXiv:1110.3193 [astro-ph.CO]

[78] R. Maartens, F. B. Abdalla, M. Jarvis, and M. G. Santos (SKA Cosmology SWG), PoS AASKA14, 016 (2015) arXiv:1501.04076 [astro-ph.CO]

[79] N. Benitez et al. (J-PAS), (2014), arXiv:1403.5237 [astro-ph.CO].

[80] S. Bonoli et al., (2020), arXiv:2007.01910 [astro-ph.CO]

[81] I. Wasserman, Phys. Rev. D 66, 123511 (2002), arXiv:astro-ph/0203137

[82] C. Rubano and P. Scudellaro, Gen. Rel. Grav. 34, 1931 (2002) arXiv:astro-ph/0203225

[83] M. Kunz, Phys. Rev. D 80, 123001 (2009) arXiv:astro-ph/0702615

[84] S. Carneiro and H. A. Borges, JCAP 06, 010 (2014) arXiv:1402.2316 [astro-ph.CO]

[85] R. von Marttens, L. Lombriser, M. Kunz, V. Marra, L. Casarini, and J. Alcaniz, Phys. Dark Univ. 28, 100490 (2020), arXiv:1911.02618 [astro-ph.CO]

[86] R. von Marttens, J. E. Gonzalez, J. Alcaniz, V. Marra, and L. Casarini, arXiv e-prints , arXiv:2011.10846 (2020), arXiv:2011.10846 [astro-ph.CO]

[87] J. M. Bardeen, Phys. Rev. D 22, 1882 (1980)

[88] C. Pigozzo, S. Carneiro, J. S. Alcaniz, H. A. Borges, and J. C. Fabris, JCAP 05, 022 (2016) arXiv:1510.01794 [astro-ph.CO].

[89] R. R. R. Reis, I. Waga, M. O. Calvao, and S. E. Joras, Phys. Rev. D 68, 061302 (2003) arXiv:astro-ph/0306004

[90] M. C. Bento, O. Bertolami, and A. A. Sen, Phys. Rev. D 70, 083519 (2004) arXiv:astro-ph/0407239.

[91] Y. Wang, D. Wands, L. Xu, J. De-Santiago, and A. Hojjati, Phys. Rev. D 87, 083503 (2013) arXiv:1301.5315 [astro-ph.CO]

[92] H. A. Borges, S. Carneiro, J. C. Fabris, and W. Zimdahl, Phys. Lett. B 727, 37 (2013) arXiv:1306.0917 [astro-ph.CO]

[93] S. Carneiro and C. Pigozzo, JCAP 10, 060 (2014), arXiv:1407.7812 [astro-ph.CO]

[94] Y. Wang, D. Wands, G.-B. Zhao, and L. Xu, Phys. Rev. D 90, 023502 (2014) arXiv:1404.5706 [astro-ph.CO]

[95] R. F. vom Marttens, L. Casarini, W. Zimdahl, W. S. Hipólito-Ricaldi, and D. F. Mota, Phys. Dark Univ. 15, 114 (2017) arXiv:1702.00651 [astro-ph.CO]

[96] R. Aurich and S. Lustig, Astropart. Phys. 97, 118 (2018), arXiv:1704.01749 [astro-ph.CO].

[97] A. Y. Kamenshchik, U. Moschella, and V. Pasquier, Phys. Lett. B 511, 265 (2001) arXiv:gr-qc/0103004

[98] J. C. Fabris, S. V. B. Goncalves, and P. E. de Souza, Gen. Rel. Grav. 34, 53 (2002) arXiv:gr-qc/0103083

[99] N. Bilic, G. B. Tupper, and R. D. Viollier, Phys. Lett. B 535, 17 (2002), arXiv:astro-ph/0111325

[100] M. C. Bento, O. Bertolami, and A. A. Sen, Phys. Rev. D 66, 043507 (2002) arXiv:gr-qc/0202064

[101] A. Dev, D. Jain, and J. S. Alcaniz, Phys. Rev. D 67, 023515 (2003) arXiv:astro-ph/0209379

[102] J. S. Alcaniz, D. Jain, and A. Dev, Phys. Rev. D 67, 043514 (2003) arXiv:astro-ph/0210476

[103] V. Gorini, A. Kamenshchik, and U. Moschella, Phys. Rev. D 67, 063509 (2003) arXiv:astro-ph/0209395

[104] H. Sandvik, M. Tegmark, M. Zaldarriaga, and I. Waga, Phys. Rev. D 69, 123524 (2004) arXiv:astro-ph/0212114

[105] V. Gorini, A. Y. Kamenshchik, U. Moschella, O. F. Piattella, and A. A. Starobinsky, JCAP 02, 016 (2008) arXiv:0711.4242 [astro-ph] 
[106] H. Velten, H. A. Borges, S. Carneiro, R. Fazolo, and S. Gomes, Mon. Not. Roy. Astron. Soc. 452, 2220 (2015) arXiv:1504.02416 [astro-ph.CO].

[107] H. A. Borges and D. Wands, Phys. Rev. D 101, 103519 (2020) arXiv:1709.08933 [astro-ph.CO]

[108] S. Carneiro and H. A. Borges, Gen. Rel. Grav. 50, 129 (2018) arXiv:1704.07825 [gr-qc].

[109] D. J. Eisenstein, H.-j. Seo, and M. J. White, Astrophys. J. 664, 660 (2007) arXiv:astro-ph/0604361.

[110] H.-J. Seo and D. J. Eisenstein, Astrophys. J. 665, 14 (2007) $\operatorname{arXiv:astro-ph/0701079.~}$

[111] A. Font-Ribera, P. McDonald, N. Mostek, B. A. Reid, H.-J. Seo, and A. Slosar, JCAP 05, 023 (2014) arXiv:1308.4164 [astro-ph.CO]

[112] M. Crocce and R. Scoccimarro, Phys. Rev. D 77, 023533 (2008), arXiv:0704.2783 [astro-ph].

[113] M. White, arXiv e-prints , arXiv:1004.0250 (2010), arXiv:1004.0250 [astro-ph.CO].

[114] L. Amendola et al., Living Rev. Rel. 21, 2 (2018) arXiv:1606.00180 [astro-ph.CO].

[115] L. Amendola and S. Tsujikawa, Dark Energy: Theory and Observations (Cambridge University Press, 2010).

[116] L. Amendola, S. Fogli, A. Guarnizo, M. Kunz, and A. Vollmer, Phys. Rev. D 89, 063538 (2014), arXiv:1311.4765 [astro-ph.CO]

[117] A. Blanchard et al. (Euclid), Astron. Astrophys. 642, A191 (2020) arXiv:1910.09273 [astro-ph.CO]

[118] L. R. Abramo and K. E. Leonard, Mon. Not. Roy. Astron. Soc. 432, 318 (2013), arXiv:1302.5444 [astro-ph.CO]

[119] G.-B. Zhao et al., Mon. Not. Roy. Astron. Soc. 457, 2377 (2016) arXiv:1510.08216 [astro-ph.CO].

[120] Y. Wang et al., Mon. Not. Roy. Astron. Soc. 409, 737 (2010), arXiv:1006.3517 [astro-ph.CO]

[121] A. Albrecht, L. Amendola, G. Bernstein, D. Clowe, D. Eisenstein, L. Guzzo, C. Hirata, D. Huterer, R. Kirshner, E. Kolb, and R. Nichol, arXiv e-prints , arXiv:0901.0721 (2009), arXiv:0901.0721 [astro-ph.IM].

[122] D. Blas, J. Lesgourgues, and T. Tram, JCAP 07, 034 (2011) arXiv:1104.2933 [astro-ph.CO]

[123] M. Aparicio Resco et al., Mon. Not. Roy. Astron. Soc. 493, 3616 (2020), arXiv:1910.02694 [astro-ph.CO].

\section{Appendix A: Fisher Matrices derivatives}

The elements of the Fisher Matrices are better visualized if we rewrite the observed matter power spectrum considering the mapping between the fiducial cosmology and the real unknown cosmological background:

$$
\mathcal{P}_{\mathrm{obs}}\left(k_{\perp}^{\mathrm{fid}}, k_{\|}^{\mathrm{fid}}, z\right)=\alpha_{\perp}^{2} \alpha_{\|}\left[b_{s}(z)+f_{s}(z)\left(\frac{k_{\|}^{2, \mathrm{fid}} \alpha_{\|}^{2}}{k_{\|}^{2, \text { fid }} \alpha_{\|}^{2}+k_{\perp}^{2, \text { fid }} \alpha_{\perp}^{2}}\right)\right]^{2} \frac{\mathcal{P}_{L, 0}\left(k=\sqrt{k_{\|}^{2, \text { fid }} \alpha_{\|}^{2}+k_{\perp}^{2, \text { fid }} \alpha_{\perp}^{2}}\right)}{\sigma_{8,0}^{2}} \exp \left(-k^{2} \mu^{2} \frac{\sigma_{z}^{2}}{\alpha_{\|}^{2} H^{2, \text { fid }}}\right)+\mathcal{P}_{\text {shot }},
$$

where, following BAO literature, we have defined the tangential and radial BAO modes as

$$
\begin{aligned}
& \alpha_{\perp}(z) \equiv \frac{r_{\perp}^{\mathrm{fid}}(z)}{r_{\perp}(z)}=\frac{D_{A}^{\mathrm{fid}}(z)}{D_{A}(z)}, \\
& \alpha_{\|}(z) \equiv \frac{r_{\|}^{\mathrm{fid}}(z)}{r_{\|}(z)}=\frac{H(z)}{H^{\mathrm{fid}}(z)},
\end{aligned}
$$

where

$$
\begin{aligned}
& r_{\|}(z)=\frac{c}{H(z) r_{s}\left(z_{d}\right)}, \\
& r_{\perp}(z)=\frac{D_{A}(z)}{r_{s}\left(z_{d}\right)},
\end{aligned}
$$

where we explicitly wrote the speed of light $\mathrm{c}$. The angular diameter distance is related to the comoving distance defined in Eq. 108 by $D_{A}(z) \equiv \chi /(1+z)$ and $r_{s}$ is the sound horizon at dragging epoch, $z_{d}$. Note that we are interested in errors on $\ln D_{A}$ and $\ln H$ which, by previous definitions, are given by

$$
\begin{aligned}
& \frac{\sigma_{\alpha_{\perp}}}{\alpha_{\perp}}=\frac{\sigma_{D_{A}}}{D_{A}}=\sigma_{\ln D_{A}}, \\
& \frac{\sigma_{\alpha_{\|}}}{\alpha_{\|}}=\frac{\sigma_{H}}{H}=\sigma_{\ln H},
\end{aligned}
$$

and considering that the Fisher Matrices have to be evaluated at the fiducial cosmology, for which we have $\alpha_{\perp}=1$ and $\alpha_{\|}=1$. We have also used the definitions

$$
\begin{aligned}
& k^{2}=k_{\|}^{2}+k_{\perp}^{2}, \\
& \mu^{2}=\frac{k_{\|}^{2}}{k^{2}},
\end{aligned}
$$


and the transformation rules

$$
\begin{aligned}
& k_{\perp}=k_{\perp}^{\mathrm{fid}} \alpha_{\perp}, \\
& k_{\|}=k_{\|}^{\mathrm{fid}} \alpha_{\|} .
\end{aligned}
$$

In the following, we report the expressions of all the functions which are needed to calculate the Fisher matrices:

- Derivatives of the power spectrum, $\ln \mathcal{P}_{\mathrm{obs}}$, with respect to $\alpha_{\perp}$ :

$$
\frac{\mathrm{d} \ln \mathcal{P}_{\mathrm{obs}}}{\mathrm{d} \alpha_{\perp}}=\left[\frac{2}{\alpha_{\perp}}+\frac{2 f_{s}}{b_{s}+f_{s} \mu^{2}} \frac{\mathrm{d} \mu^{2}}{\mathrm{~d} \alpha_{\perp}}+\frac{\mathrm{d} \ln \mathcal{P}_{L, 0}}{\mathrm{~d} k} \frac{\mathrm{d} k}{\mathrm{~d} \alpha_{\perp}}-\frac{k \sigma_{z}^{2}}{\alpha_{\|}^{2} H^{2, \mathrm{fid}}}\left(2 \mu^{2} \frac{\partial k}{\partial \alpha_{\perp}}+k \frac{\partial \mu^{2}}{\partial \alpha_{\perp}}\right)\right] \frac{\mathcal{P}_{g}}{\mathcal{P}_{\mathrm{obs}}},
$$

with

$$
\begin{aligned}
\frac{\mathrm{d} \mu^{2}}{\mathrm{~d} \alpha_{\perp}} & =-\frac{2}{\alpha_{\perp}} \mu^{2}\left(1-\mu^{2}\right), \\
\frac{\mathrm{d} k}{\mathrm{~d} \alpha_{\perp}} & =\frac{k}{\alpha_{\perp}}\left(1-\mu^{2}\right) .
\end{aligned}
$$

- Derivatives of the power spectrum, $\ln \mathcal{P}_{\mathrm{obs}}$, with respect to $\alpha_{\|}$, when we consider the baryonic matter linear contrast, $\delta_{b}$ :

$$
\frac{\mathrm{d} \ln \mathcal{P}_{\mathrm{obs}}}{\mathrm{d} \alpha_{\|}}=\left[\frac{1}{\alpha_{\|}}+\frac{2 f_{s}}{b_{s}+f_{s} \mu^{2}} \frac{\mathrm{d} \mu^{2}}{\mathrm{~d} \alpha_{\|}}+\frac{\mathrm{d} \ln \mathcal{P}_{L, 0}}{\mathrm{~d} k} \frac{\mathrm{d} k}{\mathrm{~d} \alpha_{\|}}+\frac{k \sigma_{z}^{2}}{\alpha_{\|}^{2} H^{2, \mathrm{fid}}}\left(-2 \mu^{2} \frac{\partial k}{\partial \alpha_{\|}}-k \frac{\partial \mu^{2}}{\partial \alpha_{\|}}+\frac{2 k \mu^{2}}{\alpha_{\|}}\right)\right] \frac{\mathcal{P}_{g}}{\mathcal{P}_{\mathrm{obs}}}
$$

and when we consider the total (dark plus baryonic) matter linear contrast, $\delta_{m}$ :

$$
\frac{\mathrm{d} \ln \mathcal{P}_{\mathrm{obs}}}{\mathrm{d} \alpha_{\|}}=\left[\frac{1}{\alpha_{\|}}+\frac{2}{b_{s}+f_{s} \mu^{2}}\left(f_{s} \frac{\mathrm{d} \mu^{2}}{\mathrm{~d} \alpha_{\|}}+\frac{\partial f_{s}}{\partial \alpha_{\|}} \mu^{2}\right)+\frac{\mathrm{d} \ln \mathcal{P}_{L, 0}}{\mathrm{~d} k} \frac{\mathrm{d} k}{\mathrm{~d} \alpha_{\|}}-\frac{k \sigma_{z}^{2}}{\alpha_{\|}^{2} H^{2, \mathrm{fid}}}\left(2 \mu^{2} \frac{\partial k}{\partial \alpha_{\|}}+k \frac{\partial \mu^{2}}{\partial \alpha_{\|}}-\frac{2 k \mu^{2}}{\alpha_{\|}}\right)\right] \frac{\mathcal{P}_{g}}{\mathcal{P}_{\mathrm{obs}}},
$$

with

$$
\begin{aligned}
& \frac{\mathrm{d} \mu^{2}}{\mathrm{~d} \alpha_{\|}}=\frac{2}{\alpha_{\|}} \mu^{2}\left(1-\mu^{2}\right), \\
& \frac{\mathrm{d} k}{\mathrm{~d} \alpha_{\|}}=\frac{k}{\alpha_{\|}} \mu^{2} .
\end{aligned}
$$

The growth rate of total matter relevant to the RSD measurements is given by $f_{s}=\left(f_{m}+g_{m}\right) \sigma_{8}$, where

$$
g_{m} \equiv \frac{Q}{H \rho_{m}}=\frac{\Gamma}{H}=-3 \alpha\left(1-\Omega_{m}\right)\left(\frac{H_{0}}{H}\right)^{2(\alpha+1)}=-3 \alpha\left(1-\Omega_{m}\right)\left(\frac{H_{0}}{\alpha_{\| \mid} H^{\text {fid }}}\right)^{2(\alpha+1)} .
$$

Then, we have

$$
\begin{aligned}
& \frac{\partial f_{s}}{\partial \alpha_{\|}}=\frac{\partial g_{m}}{\partial \alpha_{\|}} \sigma_{8} \\
& \frac{\partial g_{m}}{\partial \alpha_{\|}}=3 \alpha\left(1-\Omega_{m}\right) \frac{2(\alpha+1)}{\alpha_{\|}^{2 \alpha+3}}\left(\frac{H_{0}}{H^{\text {fid }}}\right)^{2(\alpha+1)} .
\end{aligned}
$$

- Derivatives of the power spectrum, $\ln \mathcal{P}_{\mathrm{obs}}$, with respect to the growth rate, $f_{s}$ :

$$
\frac{\mathrm{d} \ln \mathcal{P}_{\mathrm{obs}}}{\mathrm{d} f_{s}}=\frac{2 \mu^{2}}{b_{s}+f_{s} \mu^{2}} \frac{\mathcal{P}_{g}}{\mathcal{P}_{\mathrm{obs}}} .
$$

- Derivatives of the power spectrum, $\ln \mathcal{P}_{\text {obs }}$, with respect to the galaxy bias, $b_{s}$ :

$$
\frac{\mathrm{d} \ln \mathcal{P}_{\mathrm{obs}}}{\mathrm{d} b_{s}}=\frac{2}{b_{s}+f_{s} \mu^{2}} \frac{\mathcal{P}_{g}}{\mathcal{P}_{\mathrm{obs}}} .
$$


- Derivatives of the power spectrum, $\ln \mathcal{P}_{\text {obs }}$, with respect to the shot noise, $\mathcal{P}_{\text {shot }}$ :

$$
\frac{\mathrm{d} \ln \mathcal{P}_{\text {obs }}}{\mathrm{d} \mathcal{P}_{\text {shot }}}=\frac{1}{\mathcal{P}_{\text {obs }}},
$$

and we have assumed $\mathcal{P}_{\text {shot }}^{\text {fid }}=0$ for the fiducial model.

- Derivatives of the power spectrum, $\ln \mathcal{P}_{\text {obs }}$, with respect to $\Omega_{m}$ :

$$
\begin{aligned}
\frac{\partial \ln \mathcal{P}_{\mathrm{obs}}}{\partial \Omega_{m}} & =\frac{\mathcal{P}_{g}}{\mathcal{P}_{\mathrm{obs}}}\left[\frac{2}{\alpha_{\perp}} \frac{\partial \alpha_{\perp}}{\partial \Omega_{m}}+\frac{1}{\alpha_{\|}} \frac{\partial \alpha_{\|}}{\partial \Omega_{m}}+\frac{2}{b_{s}+f_{s} \mu^{2}}\left(\frac{\partial b_{s}}{\partial \Omega_{m}}+\frac{\partial f_{s}}{\partial \Omega_{m}} \mu^{2}+f_{s} \frac{\partial \mu^{2}}{\partial \Omega_{m}}\right)+\frac{1}{\mathcal{P}_{L, 0}} \frac{\partial \mathcal{P}_{L, 0}}{\partial \Omega_{m}}\right. \\
& \left.-\frac{k \sigma_{z}^{2}}{\alpha_{\|}^{2} H^{2, \text { fid }}}\left(2 \mu^{2} \frac{\partial k}{\partial \Omega_{m}}+k \frac{\partial \mu^{2}}{\partial \Omega_{m}}-\frac{2 k \mu^{2}}{\alpha_{\|}} \frac{\partial \alpha_{\|}}{\partial \Omega_{m}}\right)\right],
\end{aligned}
$$

with

$$
\begin{gathered}
\frac{\partial k}{\partial \Omega_{m}}=\frac{\partial k}{\partial \alpha_{\perp}} \frac{\partial \alpha_{\perp}}{\partial \Omega_{m}}+\frac{\partial k}{\partial \alpha_{\|}} \frac{\partial \alpha_{\|}}{\partial \Omega_{m}}, \\
\frac{\partial \mu^{2}}{\partial \Omega_{m}}=\frac{\partial \mu^{2}}{\partial \alpha_{\perp}} \frac{\partial \alpha_{\perp}}{\partial \Omega_{m}}+\frac{\partial \mu^{2}}{\partial \alpha_{\|}} \frac{\partial \alpha_{\|}}{\partial \Omega_{m}} .
\end{gathered}
$$

- Derivatives of the radial mode, $\alpha_{\|}$, with respect to $\Omega_{m}$ :

$$
\begin{aligned}
& \frac{\partial \alpha_{\|}}{\partial \Omega_{m}}=\frac{1}{E^{\text {fid }}} \frac{\partial E}{\partial \Omega_{m}}, \\
& \frac{\partial E}{\partial \Omega_{m}}=\frac{\left[a^{-3(\alpha+1)}-1\right]}{2(\alpha+1)} \frac{\left[\Omega_{m}\left(a^{-3(\alpha+1)}-1\right)+1\right]^{-\alpha /(\alpha+1)}}{\sqrt{\left[\Omega_{m}\left(a^{-3(\alpha+1)}-1\right)+1\right]^{1 /(\alpha+1)}}},
\end{aligned}
$$

where $E \equiv H / H_{0}$.

- Derivatives of the tangential mode, $\alpha_{\perp}$, with respect to $\Omega_{m}$ :

$$
\begin{aligned}
\frac{\partial \alpha_{\perp}}{\partial \Omega_{m}} & =-\alpha_{\perp} \frac{1}{d_{C}} \frac{\partial d_{C}}{\partial \Omega_{m}}, \\
d_{C}(z) & =\int_{0}^{z} \mathrm{~d} z^{\prime} \frac{1}{E\left(z^{\prime}\right)}, \\
\frac{\partial d_{C}}{\partial \Omega_{m}} & =-\int_{0}^{z} \mathrm{~d} z^{\prime} \frac{1}{E^{2}\left(z^{\prime}\right)} \frac{\partial E\left(z^{\prime}\right)}{\partial \Omega_{m}} .
\end{aligned}
$$

- Derivatives of the linear power spectrum, $\mathcal{P}_{L, 0}$, with respect to $\Omega_{m}$ :

$$
\frac{\partial \mathcal{P}_{L, 0}}{\partial \Omega_{m}}=\mathcal{P}_{0} n_{s} k^{n_{s}-1}\left(\frac{\partial k}{\partial \Omega_{m}}\right) \mathcal{T}^{2}+\mathcal{P}_{0} k^{n_{s}} \frac{\partial \mathcal{T}^{2}}{\partial \Omega_{m}},
$$

where derivatives of the transfer function are calculated numerically.

- Derivatives of the growth rate, $f_{s}=f_{b} \sigma_{8}$, with respect to $\Omega_{m}$ : when considering only baryonic matter,

$$
\frac{\partial f_{s}}{\partial \Omega_{m}}=\left(\frac{\partial f_{b}}{\partial \Omega_{m}}\right) \sigma_{8}+f_{b}\left(\frac{\partial \sigma_{8}}{\partial \Omega_{m}}\right) .
$$

For total matter, the growth rate relevant to the RSD measurements is given by $f_{s}=\left(f_{m}+g_{m}\right) \sigma_{8}$, where $g_{m}=Q /\left(H \rho_{m}\right)$. Then, it follows that

$$
\frac{\partial f_{s}}{\partial \Omega_{m}}=\left(\frac{\partial f_{m}}{\partial \Omega_{m}}+\frac{\partial g_{m}}{\partial \Omega_{m}}\right) \sigma_{8}+\left(f_{m}+g_{m}\right)\left(\frac{\partial \sigma_{8}}{\partial \Omega_{m}}\right),
$$


where

$$
\frac{\partial g_{m}}{\partial \Omega_{m}}=3 \alpha\left(\frac{H_{0}}{\alpha_{\|} H^{\mathrm{fid}}}\right)^{2(\alpha+1)}\left[1+\frac{2\left(1-\Omega_{m}\right)(1+\alpha)}{\alpha_{\|}} \frac{\partial \alpha_{\|}}{\partial \Omega_{m}}\right]
$$

and

$$
\frac{\partial \sigma_{8}}{\partial \Omega_{m}}=\frac{\sigma_{8,0}}{\delta_{m, 0}}\left(\frac{\partial \delta_{m}}{\partial \Omega_{m}}\right)+\sigma_{8,0}\left(-\frac{\delta_{m}}{\delta_{m, 0}^{2}}\right)\left(\frac{\partial \delta_{m, 0}}{\partial \Omega_{m}}\right) .
$$

Note that derivatives of the density contrasts $\delta_{b, m}$ and of the growth rates $f_{b, m}$ are calculated numerically.

- Derivatives of the bias factor, $b_{s}$, with respect to $\Omega_{m}$ :

$$
\frac{\partial b_{s}}{\partial \Omega_{m}}=\left(\frac{\partial b_{g}}{\partial \Omega_{m}}\right) \sigma_{8}+b_{g}\left(\frac{\partial \sigma_{8}}{\partial \Omega_{m}}\right) .
$$

Given the different definition of the galaxy bias for the tracers we have used in this work, in order to treat each of them in the same way, we have set all $\partial b_{g} / \partial \Omega_{m}=0$.

- Derivatives of the power spectrum, $\ln \mathcal{P}_{\mathrm{obs}}$, with respect to $\alpha$ : the same equations hold, but with

$$
\begin{aligned}
\frac{\partial E}{\partial \alpha} & =-\frac{\sqrt{\left[\Omega_{m}\left(a^{-3(\alpha+1)}-1\right)+1\right]^{\frac{1}{\alpha+1}}}}{2(\alpha+1)^{2}}\left[\frac{3(\alpha+1) \Omega_{m} a^{-3(\alpha+1)} \log (a)}{\Omega_{m}\left(a^{-3(\alpha+1)}-1\right)+1}+\log \left\{\Omega_{m}\left(a^{-3(\alpha+1)}-1\right)+1\right\}\right], \\
\frac{\partial g_{m}}{\partial \alpha} & =-3\left(1-\Omega_{m}\right)\left(\frac{H_{0}}{\alpha_{||} H^{\text {fid }}}\right)^{2(\alpha+1)}\left[1+2 \alpha \ln \left(\frac{H_{0}}{\alpha_{||} H^{\text {fid }}}\right)-\frac{2 \alpha(\alpha+1)}{\alpha_{\|}} \frac{\partial \alpha_{||}}{\partial \alpha}\right] .
\end{aligned}
$$

- Derivatives of the power spectrum, $\ln \mathcal{P}_{\text {obs }}$, with respect to $h$ : the same equations hold, except for:

$$
\begin{aligned}
\frac{\partial \alpha_{||}}{\partial h} & =\frac{\alpha_{||}}{h}, \\
\frac{\partial \alpha_{\perp}}{\partial h} & =\frac{\alpha_{\perp}}{h}, \\
\frac{\partial \delta_{b, m}}{\partial h} & =\frac{\partial \sigma_{8}}{\partial h}=\frac{\partial f_{b, m}}{\partial h}=\frac{\partial g_{m}}{\partial h}=0 .
\end{aligned}
$$

- Derivatives of the power spectrum, $\ln \mathcal{P}_{\text {obs }}$, with respect to $n_{s}$ : all derivatives are zero, except for:

$$
\begin{aligned}
\frac{\partial \mathcal{P}_{L, 0}}{\partial n_{s}} & =\mathcal{P}_{L, 0} \ln k, \\
\frac{\partial \ln \mathcal{P}_{\mathrm{obs}}}{\partial n_{s}} & =\frac{\mathcal{P}_{g}}{\mathcal{P}_{\mathrm{obs}}} \ln k .
\end{aligned}
$$




\section{Appendix B: Tables for BAO}

Table III. Errors for Euclid using baryons and total matter. As we are focusing only on geometrical cosmological quantities, there is no difference between the baryons and the total matter scenario in this case.

\begin{tabular}{c|cc}
\hline \hline \multicolumn{2}{|c}{ BAO } \\
\hline & \multicolumn{2}{|c}{ ELG } \\
\hline$z$ & $\sigma_{\Omega_{m}}$ & $\sigma_{\alpha}$ \\
\hline \hline 1.00 & 0.128 & 0.503 \\
1.20 & 0.105 & 0.376 \\
1.40 & 0.097 & 0.321 \\
1.65 & 0.085 & 0.260 \\
\hline Total & 0.044 & 0.148 \\
\hline \hline
\end{tabular}

Table IV. Errors for DESI using baryons and total matter. As we are focusing only on geometrical cosmological quantities, there is no difference between the baryons and the total matter scenario in this case.

\begin{tabular}{c|cc}
\hline \hline \multicolumn{2}{|c}{ BAO } \\
\hline & \multicolumn{2}{|c}{ Multi-tracers } \\
\hline$z$ & $\sigma_{\Omega_{m}}$ & $\sigma_{\alpha}$ \\
\hline \hline 0.1 & 6.101 & 163.976 \\
0.3 & 0.804 & 7.875 \\
0.5 & 0.893 & 5.533 \\
0.7 & 0.160 & 0.754 \\
0.9 & 0.114 & 0.461 \\
1.1 & 0.123 & 0.455 \\
1.3 & 0.191 & 0.944 \\
1.5 & 0.222 & 0.920 \\
1.7 & 0.356 & 1.302 \\
\hline Total & 0.051 & 0.211 \\
\hline \hline
\end{tabular}


Table V. Errors for J-PAS $4000 \mathrm{deg}^{2}$ using baryons and total matter. As we are focusing only on geometrical cosmological quantities, there is no difference between the baryons and the total matter scenario in this case.

\begin{tabular}{|c|c|c|c|c|c|c|c|c|}
\hline \multicolumn{9}{|c|}{$\mathrm{BAO}$} \\
\hline \multirow[b]{2}{*}{$z$} & \multicolumn{2}{|c|}{ Multi-tracers } & \multicolumn{2}{|c|}{ LRG } & \multicolumn{2}{|c|}{ ELG } & \multicolumn{2}{|c|}{ QSO } \\
\hline & $\sigma_{\Omega_{m}}$ & $\sigma_{\alpha}$ & $\sigma_{\Omega_{m}}$ & $\sigma_{\alpha}$ & $\sigma_{\Omega_{m}}$ & $\sigma_{\alpha}$ & $\sigma_{\Omega_{m}}$ & $\sigma_{\alpha}$ \\
\hline 0.3 & 1.301 & 11.894 & 1.529 & 15.218 & 1.415 & 13.589 & 66.832 & 625.066 \\
\hline 0.5 & 0.492 & 2.962 & 0.592 & 3.900 & 0.549 & 3.487 & 11.665 & 71.929 \\
\hline 0.7 & 0.278 & 1.317 & 0.363 & 1.879 & 0.321 & 1.603 & 4.867 & 23.517 \\
\hline 0.9 & 0.239 & 0.983 & 0.430 & 1.848 & 0.276 & 1.168 & 2.417 & 9.937 \\
\hline 1.1 & 0.304 & 1.128 & 1.797 & 6.662 & 0.335 & 1.248 & 1.457 & 5.346 \\
\hline 1.3 & 0.544 & 1.838 & - & - & 0.858 & 2.884 & 0.940 & 3.178 \\
\hline 1.5 & 0.756 & 2.403 & - & - & - & - & 0.756 & 2.403 \\
\hline 1.7 & 0.686 & 2.078 & - & - & - & - & 0.686 & 2.078 \\
\hline 1.9 & 0.638 & 1.865 & - & - & - & - & 0.638 & 1.865 \\
\hline 2.1 & 0.605 & 1.719 & - & - & - & - & 0.605 & 1.719 \\
\hline 2.3 & 0.585 & 1.626 & - & - & - & - & 0.585 & 1.626 \\
\hline 2.5 & 0.571 & 1.560 & - & - & - & - & 0.571 & 1.560 \\
\hline 2.7 & 0.564 & 1.520 & - & - & - & - & 0.564 & 1.520 \\
\hline 2.9 & 0.566 & 1.506 & - & - & - & - & 0.566 & 1.506 \\
\hline 3.1 & 0.570 & 1.505 & - & - & - & - & 0.570 & 1.505 \\
\hline 3.3 & 0.576 & 1.510 & - & - & - & - & 0.576 & 1.510 \\
\hline 3.5 & 0.588 & 1.531 & - & - & - & - & 0.588 & 1.531 \\
\hline 3.7 & 0.664 & 1.716 & - & - & - & - & 0.664 & 1.716 \\
\hline 3.9 & 0.755 & 1.939 & - & - & - & - & 0.755 & 1.939 \\
\hline Total & 0.058 & 0.220 & 0.191 & 0.945 & 0.114 & 0.488 & 0.150 & 0.425 \\
\hline
\end{tabular}


Table VI. Errors for J-PAS $8500 \mathrm{deg}^{2}$ using baryons and total matter. As we are focusing only on geometrical cosmological quantities, there is no difference between the baryons and the total matter scenario in this case.

\begin{tabular}{c|cc||cc||cc||cc}
\hline \hline \multicolumn{7}{c}{} & \multicolumn{5}{c}{ BAO } \\
\hline & Multi-tracers & \multicolumn{2}{|c}{ LRG } & \multicolumn{2}{|c}{ ELG } & \multicolumn{2}{|c}{ QSO } \\
\hline$z$ & $\sigma_{\Omega_{m}}$ & $\sigma_{\alpha}$ & $\sigma_{\Omega_{m}}$ & $\sigma_{\alpha}$ & $\sigma_{\Omega_{m}}$ & $\sigma_{\alpha}$ & $\sigma_{\Omega_{m}}$ & $\sigma_{\alpha}$ \\
\hline \hline 0.3 & 0.892 & 8.159 & 1.049 & 10.439 & 0.971 & 9.322 & 45.846 & 428.792 \\
0.5 & 0.337 & 2.032 & 0.406 & 2.675 & 0.376 & 2.392 & 8.002 & 49.343 \\
0.7 & 0.191 & 0.903 & 0.249 & 1.289 & 0.220 & 1.100 & 3.338 & 16.133 \\
0.9 & 0.164 & 0.674 & 0.295 & 1.267 & 0.189 & 0.801 & 1.658 & 6.817 \\
1.1 & 0.208 & 0.774 & 1.232 & 4.570 & 0.230 & 0.856 & 0.999 & 3.668 \\
1.3 & 0.373 & 1.261 & - & - & 0.589 & 1.978 & 0.645 & 2.180 \\
1.5 & 0.519 & 1.649 & - & - & - & - & 0.519 & 1.649 \\
1.7 & 0.470 & 1.426 & - & - & - & - & 0.470 & 1.426 \\
1.9 & 0.438 & 1.279 & - & - & - & - & 0.438 & 1.279 \\
2.1 & 0.415 & 1.179 & - & - & - & - & 0.415 & 1.179 \\
2.3 & 0.402 & 1.116 & - & - & - & - & 0.585 & 1.626 \\
2.5 & 0.392 & 1.070 & - & - & - & - & 0.392 & 1.070 \\
2.7 & 0.387 & 1.042 & - & - & - & - & 0.387 & 1.042 \\
2.9 & 0.388 & 1.033 & - & - & - & - & 0.388 & 1.033 \\
3.1 & 0.391 & 1.033 & - & - & - & - & 0.391 & 1.033 \\
3.3 & 0.395 & 1.036 & - & - & - & - & 0.395 & 1.036 \\
3.5 & 0.403 & 1.050 & - & - & - & - & 0.403 & 1.050 \\
3.7 & 0.455 & 1.177 & - & - & - & - & 0.455 & 1.177 \\
3.9 & 0.518 & 1.330 & - & - & - & - & 0.518 & 1.330 \\
\hline Total & 0.040 & 0.151 & 0.131 & 0.648 & 0.078 & 0.335 & 0.103 & 0.292 \\
\hline \hline & & & & & & & & \\
\hline
\end{tabular}




\section{Appendix C: Tables for BAO + RSD}

Table VII. Errors for Euclid using baryons and total matter.

\begin{tabular}{c|cc|cc}
\hline \hline \multicolumn{5}{c}{ BAO+RSD } \\
\hline & \multicolumn{4}{c}{ ELG } \\
& \multicolumn{3}{c}{ tot. $\mathrm{m}}$. & \multicolumn{1}{c}{ bar. $\mathrm{m}}$. \\
\hline$z$ & $\sigma_{\Omega_{m}}$ & $\sigma_{\alpha}$ & $\sigma_{\Omega_{m}}$ & $\sigma_{\alpha}$ \\
\hline \hline 1.00 & 0.040 & 0.119 & 0.10 & 0.404 \\
1.20 & 0.037 & 0.104 & 0.095 & 0.346 \\
1.40 & 0.038 & 0.099 & 0.093 & 0.312 \\
1.65 & 0.036 & 0.087 & 0.083 & 0.258 \\
\hline Total & 0.019 & 0.087 & 0.041 & 0.138 \\
\hline \hline
\end{tabular}

Table VIII. Errors for DESI using baryons and total matter.

\begin{tabular}{c|cc|cc}
\hline \hline \multicolumn{4}{c}{ BAO+RSD } \\
\multicolumn{4}{c}{ Multi-tracers } \\
& \multicolumn{3}{c}{ tot. m. } & bar. m. \\
\hline$z$ & $\sigma_{\Omega_{m}}$ & $\sigma_{\alpha}$ & $\sigma_{\Omega_{m}}$ & $\sigma_{\alpha}$ \\
\hline \hline 0.1 & 1.013 & 4.683 & 1.289 & 3.369 \\
0.3 & 0.137 & 0.470 & 0.183 & 0.632 \\
0.5 & 0.155 & 0.511 & 0.201 & 1.069 \\
0.7 & 0.038 & 0.145 & 0.084 & 0.387 \\
0.9 & 0.034 & 0.114 & 0.083 & 0.338 \\
1.1 & 0.041 & 0.120 & 0.104 & 0.392 \\
1.3 & 0.040 & 0.126 & 0.076 & 0.384 \\
1.5 & 0.054 & 0.154 & 0.132 & 0.570 \\
1.7 & 0.100 & 0.252 & 0.267 & 1.019 \\
\hline Total & 0.017 & 0.054 & 0.036 & 0.150 \\
\hline \hline & & & & \\
\hline \hline
\end{tabular}


Table IX. Errors for J-PAS $4000 \mathrm{deg}^{2}$ using baryons and total matter.

\begin{tabular}{|c|c|c|c|c|c|c|c|c|c|c|c|c|c|c|c|c|}
\hline \multicolumn{17}{|c|}{$\mathrm{BAO}+\mathrm{RSD}$} \\
\hline & \multicolumn{4}{|c|}{ Multi-tracers } & \multicolumn{4}{|c|}{ LRG } & \multicolumn{4}{|c|}{ ELG } & \multicolumn{4}{|c|}{ QSO } \\
\hline & \multicolumn{2}{|c|}{ tot. $\mathrm{m}$. } & \multicolumn{2}{|c|}{ bar. m. } & \multicolumn{2}{|c|}{ tot. $\mathrm{m}$. } & \multicolumn{2}{|c|}{ bar. m. } & \multicolumn{2}{|c|}{ tot. $\mathrm{m}$. } & \multicolumn{2}{|c|}{ bar. m. } & \multicolumn{2}{|c|}{ tot. $\mathrm{m}$. } & \multicolumn{2}{|c|}{ bar. m. } \\
\hline$z$ & $\sigma_{\Omega_{m}}$ & $\sigma_{\alpha}$ & $\sigma_{\Omega_{m}}$ & $\sigma_{\alpha}$ & $\sigma_{\Omega_{m}}$ & $\sigma_{\alpha}$ & $\sigma_{\Omega_{m}}$ & $\sigma_{\alpha}$ & $\sigma_{\Omega_{m}}$ & $\sigma_{\alpha}$ & $\sigma_{\Omega_{m}}$ & $\sigma_{\alpha}$ & $\sigma_{\Omega_{m}}$ & $\sigma_{\alpha}$ & $\sigma_{\Omega_{m}}$ & $\sigma_{\alpha}$ \\
\hline 0.3 & 0.124 & 0.753 & 0.185 & 1.030 & 0.286 & 0.894 & 0.369 & 1.198 & 0.208 & 0.836 & 0.291 & 1.126 & 6.692 & 26.666 & 9.464 & 36.166 \\
\hline 0.5 & 0.078 & 0.383 & 0.144 & 0.784 & 0.139 & 0.420 & 0.178 & 0.911 & 0.105 & 0.393 & 0.159 & 0.831 & 1.532 & 5.510 & 2.251 & 11.465 \\
\hline 0.7 & 0.064 & 0.253 & 0.144 & 0.668 & 0.105 & 0.301 & 0.176 & 0.928 & 0.079 & 0.266 & 0.155 & 0.769 & 0.856 & 2.754 & 1.590 & 7.691 \\
\hline 0.9 & 0.071 & 0.227 & 0.166 & 0.692 & 0.139 & 0.368 & 0.283 & 1.291 & 0.081 & 0.246 & 0.186 & 0.809 & 0.547 & 1.587 & 1.238 & 5.272 \\
\hline 1.1 & 0.099 & 0.279 & 0.238 & 0.913 & 0.565 & 1.375 & 1.307 & 5.107 & 0.107 & 0.300 & 0.260 & 0.998 & 0.406 & 1.081 & 1.001 & 3.820 \\
\hline 1.3 & 0.184 & 0.474 & 0.451 & 1.572 & - & - & - & - & 0.269 & 0.701 & 0.687 & 2.378 & 0.310 & 0.772 & 0.769 & 2.693 \\
\hline 1.5 & 0.282 & 0.663 & 0.679 & 2.225 & - & - & - & - & - & - & - & - & 0.282 & 0.663 & 0.679 & 2.225 \\
\hline 1.7 & 0.280 & 0.626 & 0.649 & 2.013 & - & - & - & - & - & - & - & - & 0.280 & 0.626 & 0.649 & 2.013 \\
\hline 1.9 & 0.280 & 0.601 & 0.621 & 1.847 & - & - & - & - & - & - & - & - & 0.280 & 0.601 & 0.621 & 1.847 \\
\hline 2.1 & 0.281 & 0.581 & 0.599 & 1.718 & - & - & - & - & - & - & - & - & 0.281 & 0.581 & 0.599 & 1.718 \\
\hline 2.3 & 0.285 & 0.573 & 0.584 & 1.625 & - & - & - & - & - & - & - & - & 0.285 & 0.573 & 0.584 & 1.625 \\
\hline 2.5 & 0.288 & 0.566 & 0.571 & 1.548 & - & - & - & - & - & - & - & - & 0.288 & 0.566 & 0.571 & 1.548 \\
\hline 2.7 & 0.293 & 0.564 & 0.563 & 1.490 & - & - & - & - & - & - & - & - & 0.293 & 0.564 & 0.563 & 1.490 \\
\hline 2.9 & 0.301 & 0.569 & 0.562 & 1.456 & - & - & - & - & - & - & - & - & 0.301 & 0.569 & 0.562 & 1.456 \\
\hline 3.1 & 0.310 & 0.577 & 0.563 & 1.430 & - & - & - & - & - & - & - & - & 0.310 & 0.577 & 0.563 & 1.430 \\
\hline 3.3 & 0.319 & 0.586 & 0.564 & 1.408 & - & - & - & - & - & - & - & - & 0.319 & 0.586 & 0.564 & 1.408 \\
\hline 3.5 & 0.330 & 0.601 & 0.570 & 1.401 & - & - & - & - & - & - & - & - & 0.330 & 0.601 & 0.570 & 1.401 \\
\hline 3.7 & 0.377 & 0.681 & 0.639 & 1.545 & - & - & - & - & - & - & - & - & 0.377 & 0.681 & 0.639 & 1.545 \\
\hline 3.9 & 0.434 & 0.776 & 0.720 & 1.718 & - & - & - & - & - & - & - & - & 0.434 & 0.776 & 0.720 & 1.718 \\
\hline Total & 0.030 & 0.082 & 0.049 & 0.176 & 0.068 & 0.189 & 0.104 & 0.499 & 0.043 & 0.132 & 0.081 & 0.345 & 0.077 & 0.154 & 0.134 & 0.370 \\
\hline
\end{tabular}


Table X. Errors for J-PAS $8500 \mathrm{deg}^{2}$ using baryons and total matter.

\begin{tabular}{|c|c|c|c|c|c|c|c|c|c|c|c|c|c|c|c|c|}
\hline \multicolumn{17}{|c|}{$\mathrm{BAO}+\mathrm{RSD}$} \\
\hline & \multicolumn{4}{|c|}{ Multi-tracers } & \multicolumn{4}{|c|}{ LRG } & \multicolumn{4}{|c|}{ ELG } & \multicolumn{4}{|c|}{ QSO } \\
\hline & \multicolumn{2}{|c|}{ tot. $\mathrm{m}$. } & \multicolumn{2}{|c|}{ bar. m. } & \multicolumn{2}{|c|}{ tot. $\mathrm{m}$. } & \multicolumn{2}{|c|}{ bar. m. } & \multicolumn{2}{|c|}{ tot. $\mathrm{m}$. } & \multicolumn{2}{|c|}{ bar. m. } & \multicolumn{2}{|c|}{ tot. $\mathrm{m}$. } & \multicolumn{2}{|c|}{ bar. m. } \\
\hline$z$ & $\sigma_{\Omega_{m}}$ & $\sigma_{\alpha}$ & $\sigma_{\Omega_{m}}$ & $\sigma_{\alpha}$ & $\sigma_{\Omega_{m}}$ & $\sigma_{\alpha}$ & $\sigma_{\Omega_{m}}$ & $\sigma_{\alpha}$ & $\sigma_{\Omega_{m}}$ & $\sigma_{\alpha}$ & $\sigma_{\Omega_{m}}$ & $\sigma_{\alpha}$ & $\sigma_{\Omega_{m}}$ & $\sigma_{\alpha}$ & $\sigma_{\Omega_{m}}$ & $\sigma_{\alpha}$ \\
\hline 0.3 & 0.085 & 0.516 & 0.127 & 0.706 & 0.196 & 0.613 & 0.253 & 0.822 & 0.143 & 0.574 & 0.200 & 0.772 & 4.590 & 18.293 & 6.492 & 24.810 \\
\hline 0.5 & 0.054 & 0.263 & 0.099 & 0.538 & 0.096 & 0.288 & 0.122 & 0.625 & 0.072 & 0.270 & 0.109 & 0.570 & 1.051 & 3.780 & 1.544 & 7.865 \\
\hline 0.7 & 0.044 & 0.174 & 0.099 & 0.458 & 0.072 & 0.206 & 0.121 & 0.637 & 0.054 & 0.182 & 0.106 & 0.527 & 0.587 & 1.889 & 1.091 & 5.276 \\
\hline 0.9 & 0.049 & 0.156 & 0.114 & 0.475 & 0.096 & 0.252 & 0.194 & 0.886 & 0.055 & 0.169 & 0.128 & 0.555 & 0.375 & 1.089 & 0.849 & 3.617 \\
\hline 1.1 & 0.068 & 0.191 & 0.164 & 0.626 & 0.388 & 0.943 & 0.896 & 3.503 & 0.073 & 0.206 & 0.178 & 0.685 & 0.278 & 0.742 & 0.687 & 2.621 \\
\hline 1.3 & 0.126 & 0.325 & 0.309 & 1.078 & - & - & - & - & 0.184 & 0.481 & 0.471 & 1.632 & 0.213 & 0.530 & 0.528 & 1.848 \\
\hline 1.5 & 0.194 & 0.454 & 0.466 & 1.526 & - & - & - & - & - & - & - & - & 0.194 & 0.454 & 0.466 & 1.526 \\
\hline 1.7 & 0.192 & 0.430 & 0.445 & 1.381 & - & - & - & - & - & - & - & - & 0.192 & 0.430 & 0.445 & 1.381 \\
\hline 1.9 & 0.192 & 0.412 & 0.426 & 1.267 & - & - & - & - & - & - & - & - & 0.192 & 0.412 & 0.426 & 1.267 \\
\hline 2.1 & 0.193 & 0.399 & 0.411 & 1.179 & - & - & - & - & - & - & - & - & 0.193 & 0.399 & 0.411 & 1.179 \\
\hline 2.3 & 0.195 & 0.393 & 0.401 & 1.114 & - & - & - & - & - & - & - & - & 0.195 & 0.393 & 0.401 & 1.114 \\
\hline 2.5 & 0.198 & 0.388 & 0.392 & 1.062 & - & - & - & - & - & - & - & - & 0.198 & 0.388 & 0.392 & 1.062 \\
\hline 2.7 & 0.201 & 0.387 & 0.386 & 1.022 & - & - & - & - & - & - & - & - & 0.201 & 0.387 & 0.386 & 1.022 \\
\hline 2.9 & 0.207 & 0.390 & 0.386 & 0.999 & - & - & - & - & - & - & - & - & 0.207 & 0.390 & 0.386 & 0.999 \\
\hline 3.1 & 0.213 & 0.396 & 0.386 & 0.981 & - & - & - & - & - & - & - & - & 0.213 & 0.396 & 0.386 & 0.981 \\
\hline 3.3 & 0.219 & 0.402 & 0.387 & 0.966 & - & - & - & - & - & - & - & - & 0.219 & 0.402 & 0.387 & 0.966 \\
\hline 3.5 & 0.227 & 0.412 & 0.391 & 0.961 & - & - & - & - & - & - & - & - & 0.227 & 0.412 & 0.391 & 0.961 \\
\hline 3.7 & 0.259 & 0.467 & 0.438 & 1.060 & - & - & - & - & - & - & - & - & 0.259 & 0.467 & 0.438 & 1.060 \\
\hline 3.9 & 0.298 & 0.533 & 0.494 & 1.178 & - & - & - & - & - & - & - & - & 0.298 & 0.533 & 0.494 & 1.178 \\
\hline Total & 0.021 & 0.056 & 0.034 & 0.121 & 0.046 & 0.129 & 0.071 & 0.342 & 0.030 & 0.090 & 0.055 & 0.236 & 0.053 & 0.106 & 0.092 & 0.254 \\
\hline
\end{tabular}




\section{Appendix D: Tables for BAO + RSD + PS}

Table XI. Errors for Euclid using baryons and total matter.

\begin{tabular}{c|cc|cc}
\hline \hline \multicolumn{4}{c}{ BAO+RSD+PS } \\
\hline & \multicolumn{3}{c}{ ELG } \\
& \multicolumn{3}{c}{ tot. m. } & \multicolumn{2}{c}{ bar. m. } \\
\hline$z$ & $\sigma_{\Omega_{m}}$ & $\sigma_{\alpha}$ & $\sigma_{\Omega_{m}}$ & $\sigma_{\alpha}$ \\
\hline \hline 1.00 & 0.0029 & 0.018 & 0.0023 & 0.038 \\
1.20 & 0.0027 & 0.014 & 0.0023 & 0.032 \\
1.40 & 0.0027 & 0.013 & 0.0025 & 0.028 \\
1.65 & 0.0025 & 0.011 & 0.0024 & 0.022 \\
\hline Total & 0.001 & 0.007 & 0.001 & 0.014 \\
\hline \hline
\end{tabular}

Table XII. Errors for DESI using baryons and total matter.

\begin{tabular}{c|cc|cc}
\hline \hline \multicolumn{4}{c}{ BAO+RSD+PS } \\
\hline & \multicolumn{3}{c}{ Multi-tracers } \\
& \multicolumn{2}{c}{ tot. $\mathrm{m}}$. & \multicolumn{2}{c}{ bar. $\mathrm{m}}$. \\
\hline$z$ & $\sigma_{\Omega_{m}}$ & $\sigma_{\alpha}$ & $\sigma_{\Omega_{m}}$ & $\sigma_{\alpha}$ \\
\hline \hline 0.1 & 0.014 & 0.354 & 0.014 & 0.194 \\
0.3 & 0.006 & 0.102 & 0.005 & 0.084 \\
0.5 & 0.008 & 0.101 & 0.007 & 0.127 \\
0.7 & 0.003 & 0.027 & 0.002 & 0.040 \\
0.9 & 0.003 & 0.019 & 0.002 & 0.034 \\
1.1 & 0.003 & 0.017 & 0.002 & 0.036 \\
1.3 & 0.003 & 0.021 & 0.002 & 0.039 \\
1.5 & 0.004 & 0.023 & 0.003 & 0.057 \\
1.7 & 0.006 & 0.036 & 0.007 & 0.101 \\
\hline Total & 0.001 & 0.009 & 0.001 & 0.016 \\
\hline \hline & & & & \\
\hline \hline
\end{tabular}


Table XIII. Errors for J-PAS $4000 \mathrm{deg}^{2}$ using baryons and total matter.

\begin{tabular}{|c|c|c|c|c|c|c|c|c|c|c|c|c|c|c|c|c|}
\hline \multicolumn{17}{|c|}{$\mathrm{BAO}+\mathrm{RSD}+\mathrm{PS}$} \\
\hline & \multicolumn{4}{|c|}{ Multi-tracers } & \multicolumn{4}{|c|}{ LRG } & \multicolumn{4}{|c|}{ ELG } & \multicolumn{4}{|c|}{ QSO } \\
\hline & \multicolumn{2}{|c|}{ tot. $\mathrm{m}$. } & \multicolumn{2}{|c|}{ bar. m. } & \multicolumn{2}{|c|}{ tot. $\mathrm{m}$. } & \multicolumn{2}{|c|}{ bar. m. } & \multicolumn{2}{|c|}{ tot. $\mathrm{m}$. } & \multicolumn{2}{|c|}{ bar. m. } & \multicolumn{2}{|c|}{ tot. $\mathrm{m}$. } & \multicolumn{2}{|c|}{ bar. m. } \\
\hline$z$ & $\sigma_{\Omega_{m}}$ & $\sigma_{\alpha}$ & $\sigma_{\Omega_{m}}$ & $\sigma_{\alpha}$ & $\sigma_{\Omega_{m}}$ & $\sigma_{\alpha}$ & $\sigma_{\Omega_{m}}$ & $\sigma_{\alpha}$ & $\sigma_{\Omega_{m}}$ & $\sigma_{\alpha}$ & $\sigma_{\Omega_{m}}$ & $\sigma_{\alpha}$ & $\sigma_{\Omega_{m}}$ & $\sigma_{\alpha}$ & $\sigma_{\Omega_{m}}$ & $\sigma_{\alpha}$ \\
\hline 0.3 & 0.010 & 0.141 & 0.009 & 0.099 & 0.011 & 0.219 & 0.010 & 0.188 & 0.011 & 0.156 & 0.010 & 0.118 & 0.272 & 3.801 & 0.256 & 2.844 \\
\hline 0.5 & 0.006 & 0.073 & 0.006 & 0.069 & 0.007 & 0.103 & 0.007 & 0.147 & 0.007 & 0.077 & 0.006 & 0.083 & 0.080 & 0.870 & 0.071 & 0.918 \\
\hline 0.7 & 0.005 & 0.047 & 0.004 & 0.060 & 0.006 & 0.066 & 0.006 & 0.139 & 0.006 & 0.049 & 0.005 & 0.071 & 0.047 & 0.421 & 0.041 & 0.622 \\
\hline 0.9 & 0.005 & 0.039 & 0.004 & 0.068 & 0.007 & 0.070 & 0.009 & 0.166 & 0.006 & 0.040 & 0.005 & 0.074 & 0.030 & 0.234 & 0.027 & 0.460 \\
\hline 1.1 & 0.007 & 0.041 & 0.006 & 0.086 & 0.026 & 0.245 & 0.033 & 0.589 & 0.007 & 0.043 & 0.006 & 0.090 & 0.022 & 0.155 & 0.023 & 0.358 \\
\hline 1.3 & 0.011 & 0.063 & 0.011 & 0.144 & - & - & - & - & 0.016 & 0.088 & 0.015 & 0.201 & 0.017 & 0.109 & 0.019 & 0.256 \\
\hline 1.5 & 0.015 & 0.093 & 0.017 & 0.205 & - & - & - & - & - & - & - & - & 0.015 & 0.093 & 0.017 & 0.205 \\
\hline 1.7 & 0.014 & 0.088 & 0.017 & 0.179 & - & - & - & - & - & - & - & - & 0.014 & 0.088 & 0.017 & 0.179 \\
\hline 1.9 & 0.014 & 0.086 & 0.016 & 0.159 & - & - & - & - & - & - & - & - & 0.014 & 0.086 & 0.016 & 0.159 \\
\hline 2.1 & 0.014 & 0.084 & 0.016 & 0.145 & - & - & - & - & - & - & - & - & 0.014 & 0.084 & 0.016 & 0.145 \\
\hline 2.3 & 0.014 & 0.084 & 0.016 & 0.135 & - & - & - & - & - & - & - & - & 0.014 & 0.084 & 0.016 & 0.135 \\
\hline 2.5 & 0.014 & 0.085 & 0.016 & 0.128 & - & - & - & - & - & - & - & - & 0.014 & 0.085 & 0.016 & 0.128 \\
\hline 2.7 & 0.014 & 0.086 & 0.016 & 0.124 & - & - & - & - & - & - & - & - & 0.014 & 0.086 & 0.016 & 0.124 \\
\hline 2.9 & 0.015 & 0.087 & 0.016 & 0.121 & - & - & - & - & - & - & - & - & 0.015 & 0.087 & 0.016 & 0.121 \\
\hline 3.1 & 0.015 & 0.090 & 0.016 & 0.120 & - & - & - & - & - & - & - & - & 0.015 & 0.090 & 0.016 & 0.120 \\
\hline 3.3 & 0.015 & 0.092 & 0.017 & 0.119 & - & - & - & - & - & - & - & - & 0.015 & 0.092 & 0.017 & 0.119 \\
\hline 3.5 & 0.016 & 0.095 & 0.017 & 0.120 & - & - & - & - & - & - & - & - & 0.016 & 0.095 & 0.017 & 0.120 \\
\hline 3.7 & 0.018 & 0.108 & 0.019 & 0.134 & - & - & - & - & - & - & - & - & 0.018 & 0.108 & 0.019 & 0.134 \\
\hline 3.9 & 0.020 & 0.124 & 0.022 & 0.150 & - & - & - & - & - & - & - & - & 0.020 & 0.124 & 0.022 & 0.150 \\
\hline Total & 0.002 & 0.016 & 0.004 & 0.038 & 0.004 & 0.042 & 0.004 & 0.075 & 0.003 & 0.023 & 0.002 & 0.035 & 0.004 & 0.024 & 0.004 & 0.037 \\
\hline
\end{tabular}


Table XIV. Errors for J-PAS $8500 \mathrm{deg}^{2}$ using baryons and total matter.

\begin{tabular}{|c|c|c|c|c|c|c|c|c|c|c|c|c|c|c|c|c|}
\hline \multicolumn{17}{|c|}{$\mathrm{BAO}+\mathrm{RSD}+\mathrm{PS}$} \\
\hline & \multicolumn{4}{|c|}{ Multi-tracers } & \multicolumn{4}{|c|}{ LRG } & \multicolumn{4}{|c|}{ ELG } & \multicolumn{4}{|c|}{ QSO } \\
\hline & \multicolumn{2}{|c|}{ tot. $\mathrm{m}$. } & \multicolumn{2}{|c|}{ bar. m. } & \multicolumn{2}{|c|}{ tot. $\mathrm{m}$. } & \multicolumn{2}{|c|}{ bar. m. } & \multicolumn{2}{|c|}{ tot. $\mathrm{m}$. } & \multicolumn{2}{|c|}{ bar. m. } & \multicolumn{2}{|c|}{ tot. $\mathrm{m}$. } & \multicolumn{2}{|c|}{ bar. m. } \\
\hline$z$ & $\sigma_{\Omega_{m}}$ & $\sigma_{\alpha}$ & $\sigma_{\Omega_{m}}$ & $\sigma_{\alpha}$ & $\sigma_{\Omega_{m}}$ & $\sigma_{\alpha}$ & $\sigma_{\Omega_{m}}$ & $\sigma_{\alpha}$ & $\sigma_{\Omega_{m}}$ & $\sigma_{\alpha}$ & $\sigma_{\Omega_{m}}$ & $\sigma_{\alpha}$ & $\sigma_{\Omega_{m}}$ & $\sigma_{\alpha}$ & $\sigma_{\Omega_{m}}$ & $\sigma_{\alpha}$ \\
\hline 0.3 & 0.007 & 0.097 & 0.006 & 0.068 & 0.007 & 0.151 & 0.007 & 0.129 & 0.007 & 0.107 & 0.007 & 0.081 & 0.186 & 2.608 & 0.176 & 1.951 \\
\hline 0.5 & 0.004 & 0.050 & 0.004 & 0.047 & 0.005 & 0.071 & 0.005 & 0.101 & 0.005 & 0.053 & 0.004 & 0.057 & 0.055 & 0.597 & 0.049 & 0.630 \\
\hline 0.7 & 0.004 & 0.032 & 0.003 & 0.041 & 0.004 & 0.045 & 0.004 & 0.095 & 0.004 & 0.033 & 0.003 & 0.049 & 0.032 & 0.289 & 0.028 & 0.427 \\
\hline 0.9 & 0.004 & 0.026 & 0.003 & 0.046 & 0.005 & 0.048 & 0.006 & 0.114 & 0.004 & 0.027 & 0.003 & 0.050 & 0.020 & 0.161 & 0.019 & 0.315 \\
\hline 1.1 & 0.005 & 0.028 & 0.004 & 0.059 & 0.018 & 0.168 & 0.022 & 0.404 & 0.005 & 0.029 & 0.004 & 0.062 & 0.015 & 0.106 & 0.015 & 0.246 \\
\hline 1.3 & 0.007 & 0.043 & 0.007 & 0.099 & - & - & - & - & 0.011 & 0.060 & 0.010 & 0.138 & 0.011 & 0.074 & 0.013 & 0.176 \\
\hline 1.5 & 0.010 & 0.064 & 0.012 & 0.141 & - & - & - & - & - & - & - & - & 0.010 & 0.064 & 0.012 & 0.141 \\
\hline 1.7 & 0.010 & 0.061 & 0.012 & 0.123 & - & - & - & - & - & - & - & - & 0.010 & 0.061 & 0.012 & 0.123 \\
\hline 1.9 & 0.010 & 0.059 & 0.011 & 0.109 & - & - & - & - & - & - & - & - & 0.010 & 0.059 & 0.011 & 0.109 \\
\hline 2.1 & 0.010 & 0.058 & 0.011 & 0.099 & - & - & - & - & - & - & - & - & 0.010 & 0.058 & 0.011 & 0.099 \\
\hline 2.3 & 0.010 & 0.058 & 0.011 & 0.093 & - & - & - & - & - & - & - & - & 0.010 & 0.058 & 0.011 & 0.093 \\
\hline 2.5 & 0.010 & 0.058 & 0.011 & 0.088 & - & - & - & - & - & - & - & - & 0.010 & 0.058 & 0.011 & 0.088 \\
\hline 2.7 & 0.010 & 0.059 & 0.011 & 0.085 & - & - & - & - & - & - & - & - & 0.010 & 0.059 & 0.011 & 0.085 \\
\hline 2.9 & 0.010 & 0.060 & 0.011 & 0.083 & - & - & - & - & - & - & - & - & 0.010 & 0.060 & 0.011 & 0.083 \\
\hline 3.1 & 0.010 & 0.062 & 0.011 & 0.082 & - & - & - & - & - & - & - & - & 0.010 & 0.062 & 0.011 & 0.082 \\
\hline 3.3 & 0.010 & 0.063 & 0.011 & 0.082 & - & - & - & - & - & - & - & - & 0.010 & 0.063 & 0.011 & 0.082 \\
\hline 3.5 & 0.011 & 0.065 & 0.012 & 0.082 & - & - & - & - & - & - & - & - & 0.011 & 0.065 & 0.012 & 0.082 \\
\hline 3.7 & 0.012 & 0.074 & 0.013 & 0.092 & - & - & - & - & - & - & - & - & 0.012 & 0.074 & 0.013 & 0.092 \\
\hline 3.9 & 0.014 & 0.085 & 0.015 & 0.103 & - & - & - & - & - & - & - & - & 0.014 & 0.085 & 0.015 & 0.103 \\
\hline Total & 0.001 & 0.011 & 0.001 & 0.013 & 0.003 & 0.029 & 0.003 & 0.051 & 0.002 & 0.015 & 0.002 & 0.024 & 0.003 & 0.017 & 0.003 & 0.025 \\
\hline
\end{tabular}

